Supporting information for manuscript

entitled

\title{
Insight into Antioxidant and Photo-protective Properties of Natural Compounds from Marine Fungus
}

\author{
Duy Quang Dao 1,,, Thi Thu Trang Phan², Thi Le Anh Nguyen 1,, \\ Hoai Trinh ${ }^{3}$, Thi Thanh Van Tran ${ }^{3}$, Jong Seok Lee ${ }^{4}$, Hee Jae Shin ${ }^{4}$, \\ Byeoung-Kyu Choi ${ }^{4}$ \\ ${ }^{1}$ Institute of Research and Development, Duy Tan University, 03 Quang Trung, Da Nang, \\ 550000, Vietnam \\ ${ }^{2}$ Faculty of Pharmacy, Duy Tan University, 03 Quang Trung, Da Nang, 550000, Vietnam \\ ${ }^{3}$ Nha Trang Institute of Technology Research and Application, Vietnam Academy of Science \\ and Technology, 02 Hung Vuong, Nha Trang, Vietnam \\ ${ }^{4}$ Korea Institute of Ocean Science and Technology, 385, Haeyang-ro, Yeongdo-gu, Busan \\ Metropolitan City 49111, Korea
}

*Corresponding authors: daoduyquang@duytan.edu.vn (Duy Quang Dao)

nguyenthileanh@duytan.edu.vn (Thi Le Anh Nguyen) 


\section{List of figures}

Figure S1: UV-Vis spectra of C1: (black) experimental, (red) CAMB3LYP/6-311+G(d,p), (magenta) PBE0/6-311+G(d,p), (blue) M05-2X/6-311+G(d,p) and olive M05-2X/6-31+G(d).

Figure S2: Structure of 2,2-diphenyl-1-picrylhydrazyl (DPPH•) (top) and 2,2'-azino-bis(3ethylbenzothiazoline-6-sulfonic acid) $\left(\mathrm{ABTS}^{\bullet+}\right)$ (bottom) radicals.

Figure S3: Optimized structures, HOMO and LUMO distributions and electrostatistic potential (ESP) maps of the five studied compounds C1-C5 calculated at the M05-2X/6-31+G(d).

Figure S4: Optimized structure and singly occupied molecular orbitals (SOMO) and SOMO-1 distributions of the transition state (TS) for the first-HAT reaction towards $\mathrm{HOO}^{-}$radical calculated at the M05-2X/6-311++G(d,p) level of theory in the gas phase. Bond lengths are in $\AA$ and angles in degree. Iso-density value is equal to $0.02 \mathrm{au}$. Values in parentheses consist in imaginary frequencies of TS.

Figure S5: Optimized structure of the transition state (TS) for the first-HAT reaction towards $\mathrm{HOO}$ - radical calculated at the M05-2X/6-311++G(d,p) level of theory in water. Bond lengths are in $\AA$ and angles in degree. Values in parentheses consist in imaginary frequencies of TS.

Figure S6: Optimized structure of the transition state (TS) for the first-HAT reaction towards $\mathrm{HOO}$ - radical calculated at the M05-2X/6-311++G(d,p) level of theory in PEA. Bond lengths are in $\AA$ and angles in degree. Values in parentheses consist in imaginary frequencies of TS

Figure S7: Optimized structures, singly occupied molecular orbital (SOMO) and SOMO-1 orbital distributions of the transition states (TS) of RAF reaction between $\mathbf{C 1}-\mathbf{C 5}$ with $\mathrm{HOO} \cdot$ radical at different positions. Bond lengths are in $\AA$ and angles in degree. The imaginary frequencies $(f)$ are also noted below each TS structure. All calculations were performed at the M05-2X/6$311++G(d, p)$ level of theory in the gas phase.

Figure S8: Internal Reaction Coordinates (IRCs) of H-atom transfer (HAT) reactions calculated at the $M 05-2 X / 6-311++G(d, p)$ in the gas phase.

Figure S9: Internal Reaction Coordinates (IRCs) of Radical Adduct Formation (RAF) reactions calculated at the M05-2X/6-311++G(d,p) in the gas phase.

Figure S10: Optimized structure of the transition states (TSs) for RAF mechanism with HOO• radical in the water. Bond lengths are in $\AA$ and angles in degree. All calculations were performed in the gas phase at the $M 05-2 X / 6-311++G(d, p)$ level of theory.

Figure S11: Optimized structure of the transition states (TSs) for RAF mechanism with HOO• radical in the PEA. Bond lengths are in $\AA$ and angles in degree. All calculations were performed in the gas phase at the $M 05-2 X / 6-311++G(d, p)$ level of theory. 


\section{List of tables}

Table S1: BDE values (in $\mathrm{kcal} \mathrm{mol}^{-1}$ ) characterizing two steps of double HT mechanism calculated in the gas phase, water and pentyl ethanoate (PEA) at the M05-2X/6-311++G(d,p)// M05-2X/6-31+G(d) level of theory.

Table S2: Reaction enthalpies $\left(\Delta_{r} H^{0}\right)$ and standard Gibbs free energies $\left(\Delta_{r} G^{0}\right)$ (in kcal mol-1) of RAF reactions between $\mathrm{C} 1-\mathrm{C} 5$ and $\mathrm{HOO}$ radical calculated at the M05-2X/6-311++G(d,p) level of theory in the gas phase, water and pentyl ethanoate (PEA).

Table S3: Intrinsic properties characterizing for double SET-PT process (IP and PDE, in kcal $\mathrm{mol}^{-1}$ ) calculated in the gas phase, water and PEA using the M05-2X/6-311++G(d,p)//M05-2X/6$31+G(d)$ level of theory.

Table S4: Intrinsic properties characterizing for double SPL-ET process (PA and ETE, in kcal $\mathrm{mol}^{-1}$ ) calculated in the gas phase, water and PEA solvent using the M05-2X/6-311++G(d,p)// M05-2X/6-31+G(d) level of theory.

Table S5: Excited energies $(\mathrm{eV})$, absorption wavelength $(\mathrm{nm})$, oscillator strength $(f)$ and transition interpretation for some lowest excited states of compounds C1-C5 Calculated by TDDFT at the M05-2X/6-31+G(d) Level of Theory in Water. 


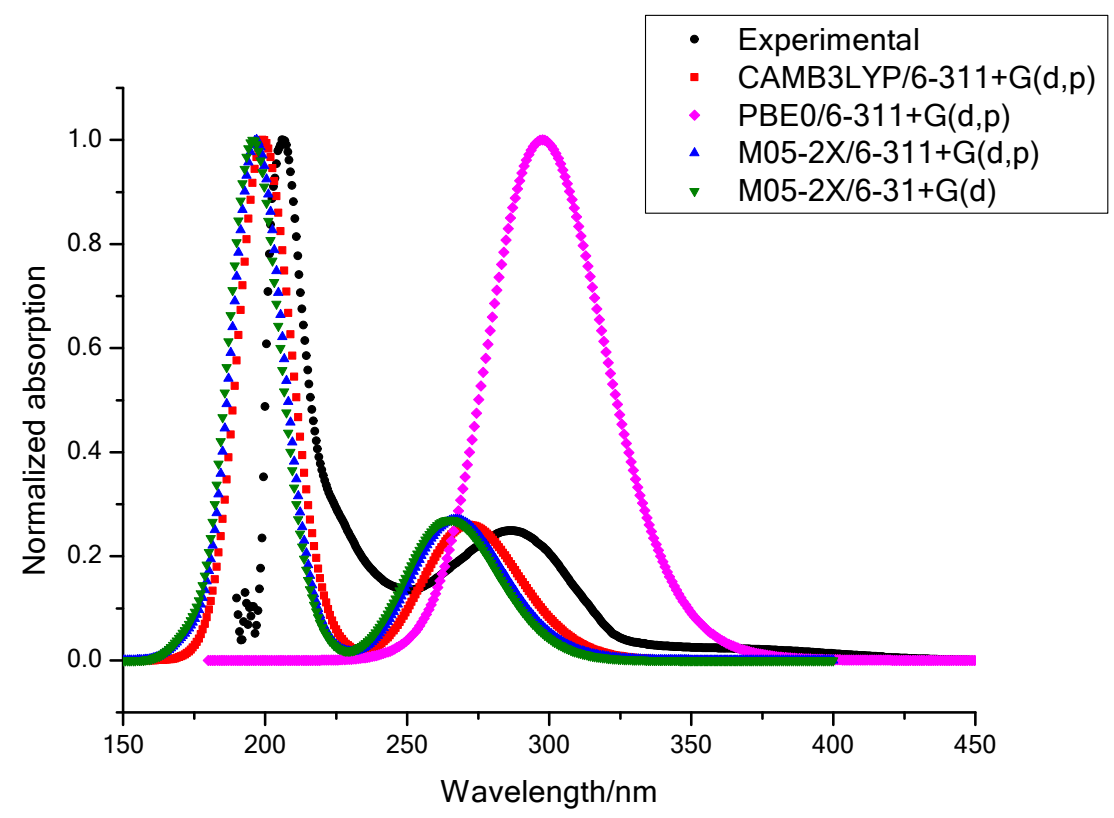

Figure S1: UV-Vis spectra of C1: (black) experimental, (red) CAMB3LYP/6-311+G(d,p), (magenta) PBE0/6-311+G(d,p), (blue) M05-2X/6-311+G(d,p) and olive M05-2X/6-31+G(d).

The choice of functional and basis-sets were carefully considered. As recommend by previous studied (see references 1-12 below), the most accurate calculation of excitation of organic molecules are performed with hybrid functionals containing an exact exchange contribution such as exchange-correlation functional CAM-B3LYP $(19 \% \mathrm{HF}+81 \% \mathrm{~B} 88$ at short-range and $65 \% \mathrm{HF}+35 \% \mathrm{~B} 88$ at long-range), PBE0 functional $(25 \% \mathrm{HF}+75 \% \mathrm{PBE}$ energy with full-range PBE correlation) and M05-2X functional (global hybrid functional with $56 \% \mathrm{HF}$ exchange). The figure below shows the electronic spectra of $\mathbf{C} 1$ performed with CAMB3LYP/6-311+G(d,p), $P B E 0 / 6-311+G(d, p)$, M05-2X/6-311+G(d,p) and M05-2X/6-31+G(d). As we can see the CAMB3LYP and M05-2X provide results in agreement with the experimental. Although the CAMB3LYP is slightly better than the M05-2X, there is no significant difference between the two functionals. Therefore, we choose the TD-M05-2X functional, which is in-line with our calculations of electronic structures and antioxidant properties.

Comparing the M05-2X/6-311+G(d,p) and M05-2X/6-31+G(d), we see almost no difference. Therefore we choose the M05-2X/6-31+G(d) level of theory for all other calculations. This also helps to balance the computed time and the accuracy.

\section{$\underline{\text { References: }}$}

1. Adamo, C.; Jacquemin, D. The calculations of excited-states properties with TimeDependent Density Functional Theory. Chem. Soc. Rev. 2013, 42, 845-856. 
2. Jacquemin, D.; Warthelet, V.; Perpète, E. A.; Adamo, C. Extensive TD-DFT Benchmark: Singlet-Excited States of Organic Molecules J. Chem. Theory. Comput. 2009, 5, 24202435.

3. Zhao, Y.; Truhlar, D. G. The M06 suite of density functionals for main group thermochemistry, thermochemical kinetics, noncovalent interactions, excited states, and transition elements: two new functionals and systematic testing of four M06-class functionals and 12 other functionals Theor. Chem. Account, 2008, 120, 215-241.

4. Caricato, M.; Trucks, G. W.; Frisch, M. J.; Wiberg, K. B. Oscillator Strength: How Does TDDFT Compare to EOM-CCSD? J. Chem. Theory. Comput. 2011, 7, 456-466.

5. Maier, T. M.; Bahmann, H.; Arbuznikov, A. V.; Kaupp, M. Validation of local hybrid functionals for TDDFT calculations of electronic excitation energies J. Chem. Phys. 2016, 144, 074106.

6. Santoro, F.; Barone, V.Lami, A.; Improta, R. The excited electronic states of adenineguanine stacked dimers in aqueous solution: a PCM/TD-DFT study. Phys. Chem. Chem. Phys. 2010, 12, 4934-4948.

7. Ristilä, M.; Matxain, J. M.; Strid, A.; Eriksson, L. A. pH-Dependent Electronic and Spectroscopic Properties of Pyridoxine (Vitamin B6). J. Phys. Chem. B. 2006, 110, 16774-16780.

8. Musa, K. A. K.; Eriksson, L. A. Photodegradation mechanism of the common non-steroid anti-inflammatory drug diclofenac and its carbazole photoproduct. Phys. Chem. Chem. Phys. 2009, 11, 4601-4610.

9. Oltean, M.; Calborean, A.; Mile, G.; Vidrigin, M.; losin, M.; Leopold, L.; Maniu, D.; Leopold, N.; Chiş. Absorption spectra of PTCDI: A combined UV-Vis and TD-DFT study. Spectrochim. Acta Part A. Mol. Biomol. Spectroscopy 2012, 97, 713-710.

10. Latouche, C.; Skouteris, D.; Palazzetti, F.; Barone, V. TD-DFT Benchmark on Inorganic $\mathrm{Pt}(\mathrm{II})$ and Ir(III) Complexes J. Chem. Theory. Comput. 2015, 11, 3281-3289.

11. Das, S.; Karmakar, S.; Saha, D.; Baitalik, S. A Combined Experimental and DFT/TDDFT Investigation of Structural, Electronic, and Cation-Induced Switching of Photophysical Properties of Bimetallic Ru(II) and Os(II) Complexes Derived from Imidazole-4,5-Dicarboxylic Acid and 2,2'-Bipyridine. Inorg. Chem. 2013, 52, 6860-6879.

12. Li, X.-N.; Wu, Z.-J.; Si, Z.-J. ; Zhang, H.-J. ; Liu, X.-J. Injection, Transport, Absorption and Phosphorescence Properties of a Series of Blue-Emitting $\operatorname{Ir}(I I I)$ Emitters in OLEDs: a DFT and Time-Dependent DFT Study. Inorg. Chem. 2009, 48, 7740-7749. 

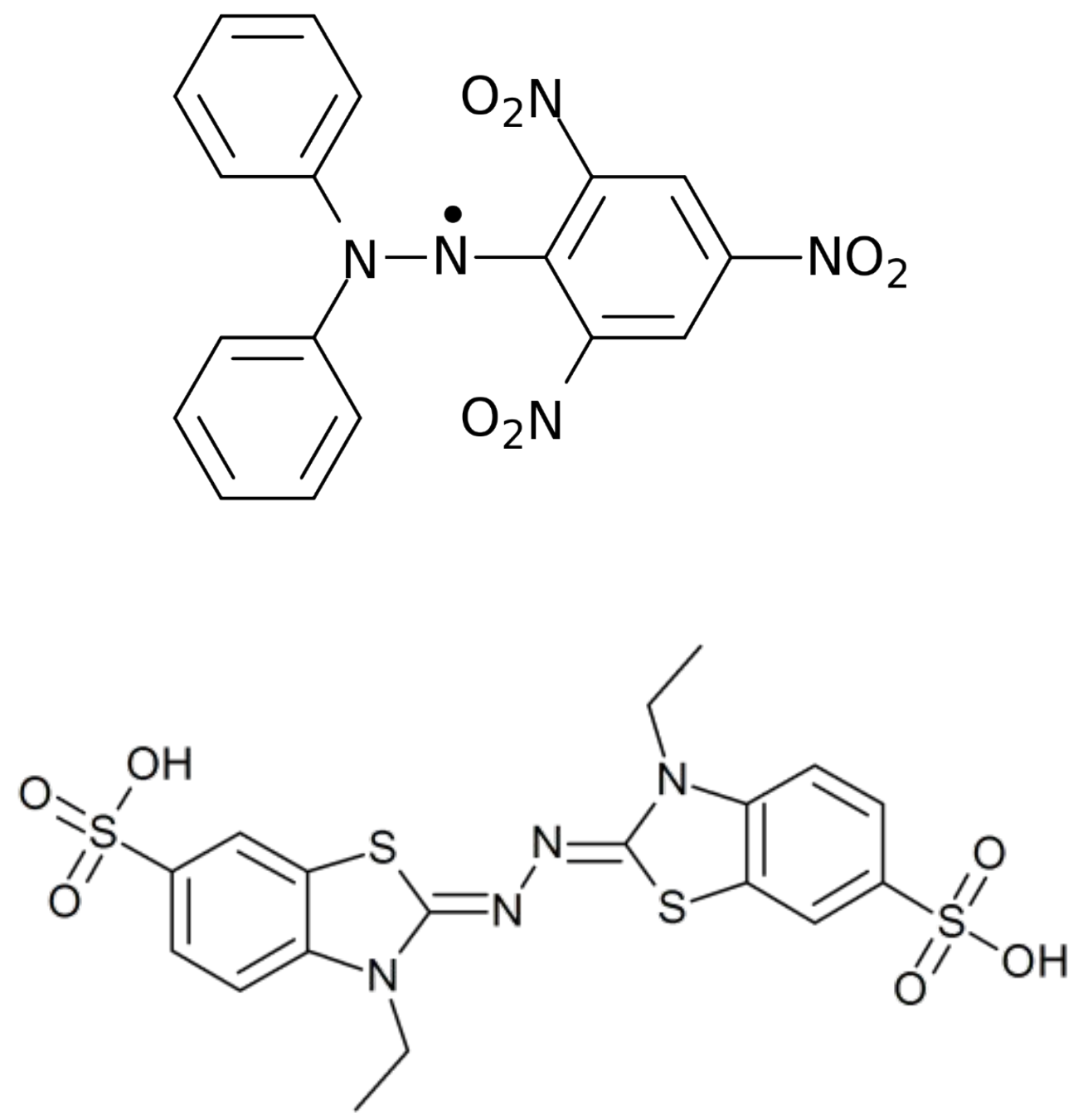

Figure S2: Structure of 2,2-diphenyl-1-picrylhydrazyl (DPPH*) (top) and 2,2' -azino-bis(3ethylbenzothiazoline-6-sulfonic acid) (ABTS•+) (bottom) radicals 


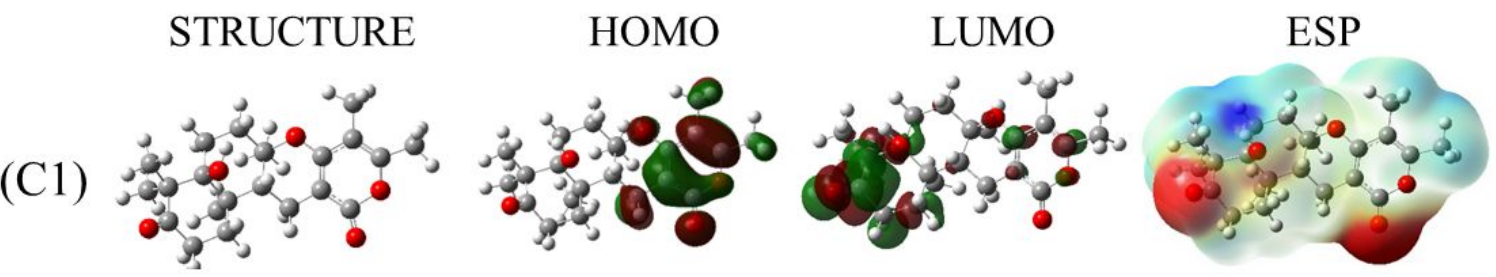

(C2) -

(C3)
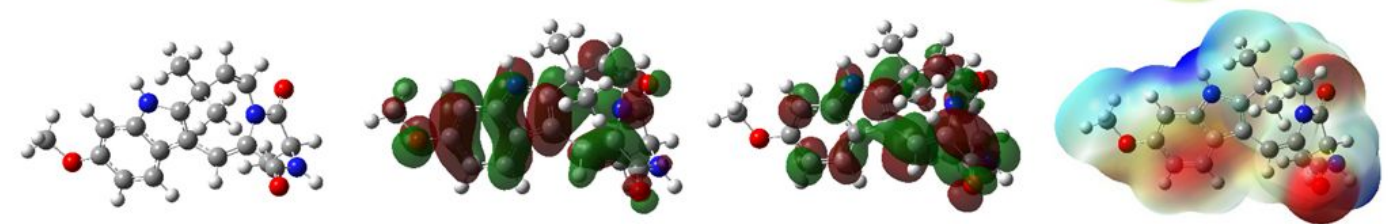

(C4)
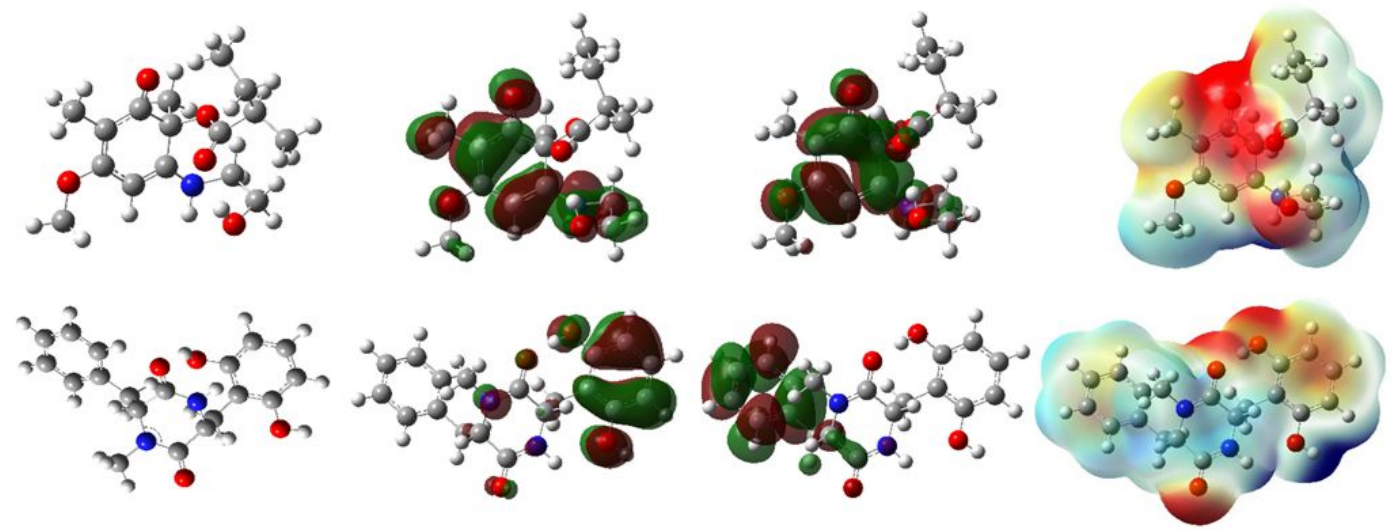

Figure S3: Optimized structures, HOMO and LUMO distributions and electrostatic potential (ESP) maps of the five studied compounds C1-C5 calculated at the M05-2X/6-31+G(d). 

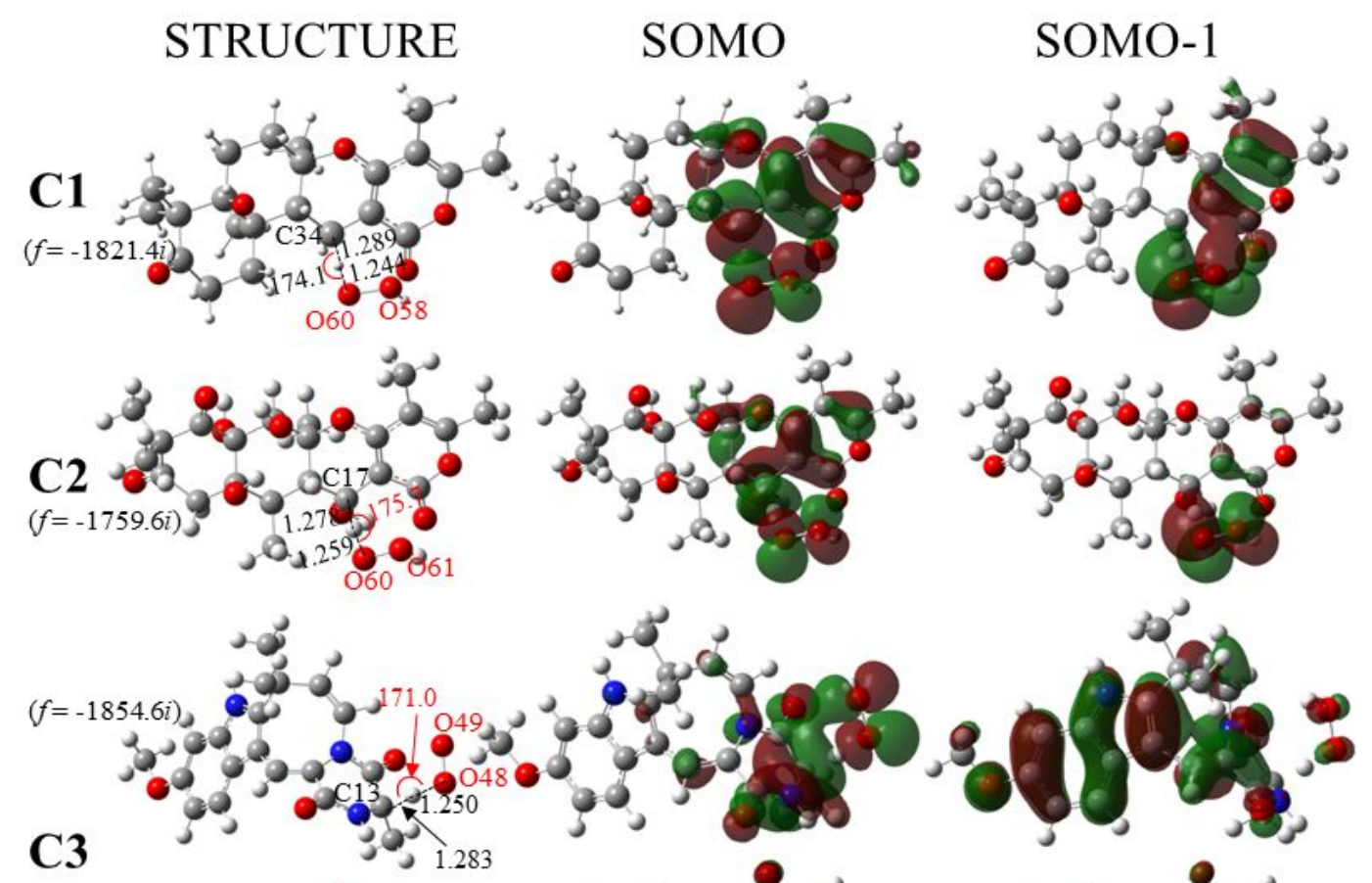

\section{C3}
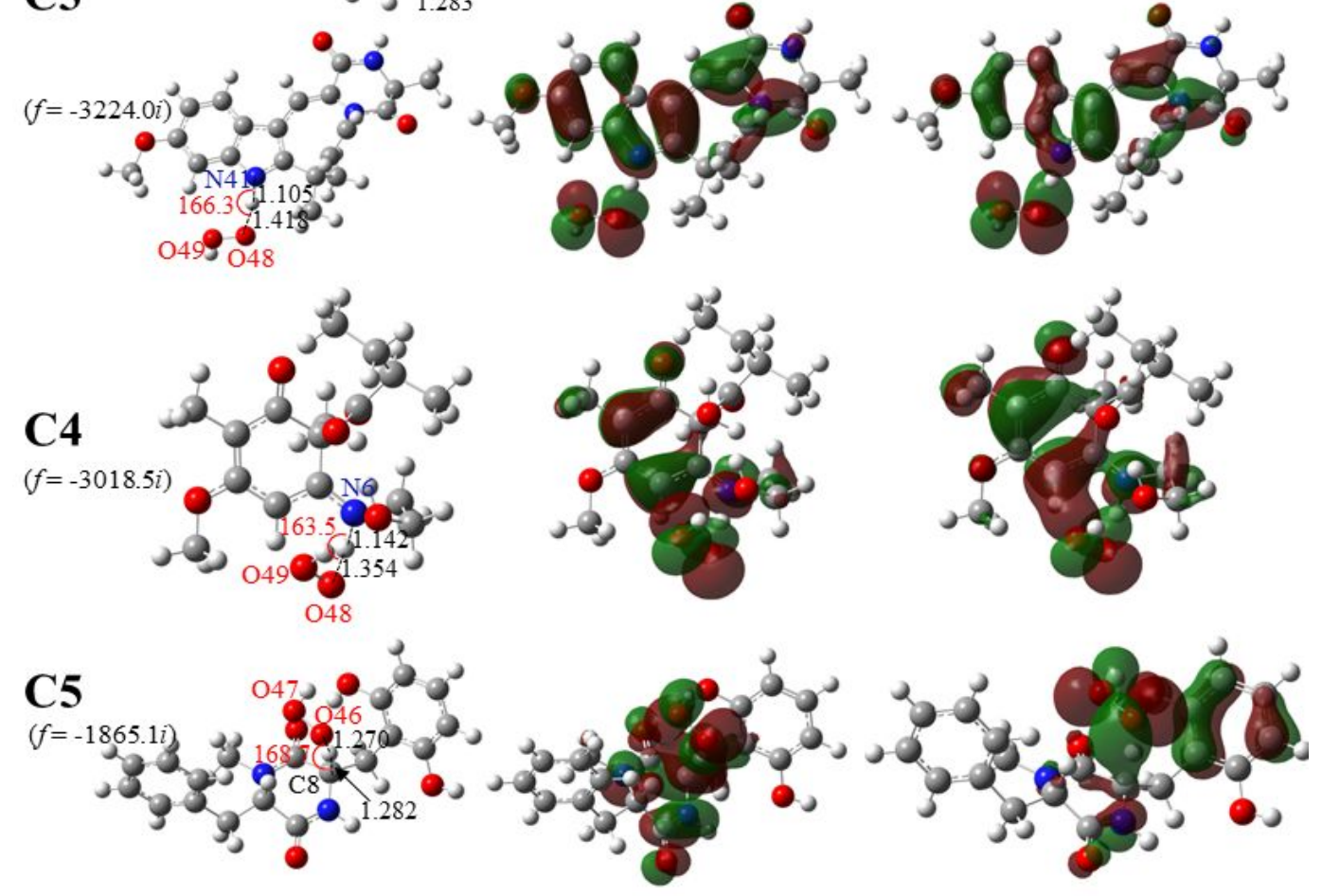

Figure S4: Optimized structure and singly occupied molecular orbitals (SOMO) and SOMO-1 distributions of the transition state (TS) for the first-HAT reaction towards $\mathrm{HOO}$ radical calculated at the M05-2X/6-311++G(d,p) level of theory in the gas phase. Bond lengths are in $\AA$ and angles in degree. Iso-density value is equal to $0.02 \mathrm{au}$. Values in parentheses consist in imaginary frequencies of TS. 

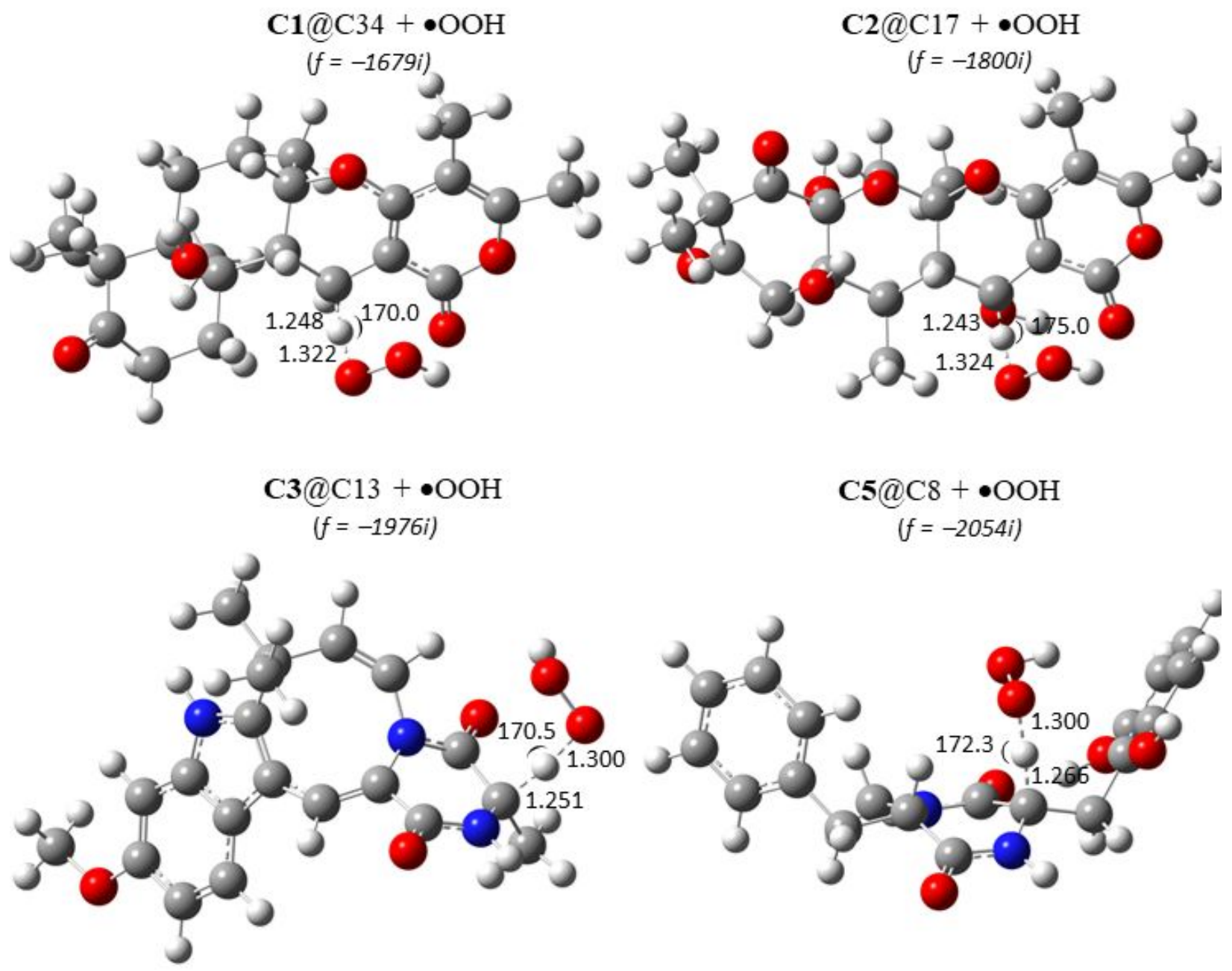
C5@C8+• $\mathrm{OOH}$
$(f=-2054 i)$

Figure S5: Optimized structure of the transition state (TS) for the first-HAT reaction towards $\mathrm{HOO} \cdot$ radical calculated at the M05-2X/6-311++G(d,p) level of theory in water. Bond lengths are in $\AA$ and angles in degree. Values in parentheses consist in imaginary frequencies of TS. 

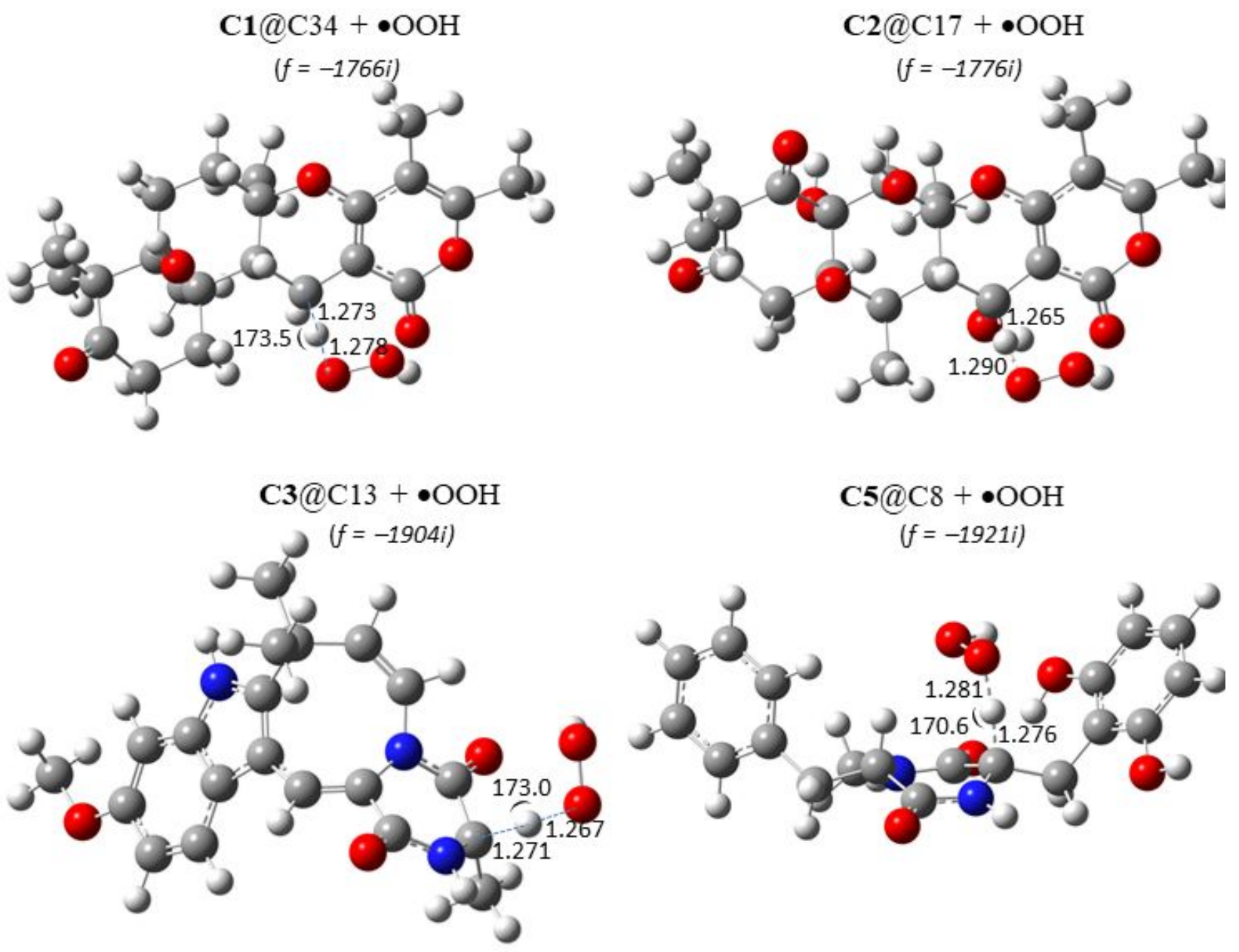

$$
\begin{gathered}
\mathrm{C5} @ \mathrm{C} 8+\bullet \mathrm{OOH} \\
(f=-1921 i)
\end{gathered}
$$

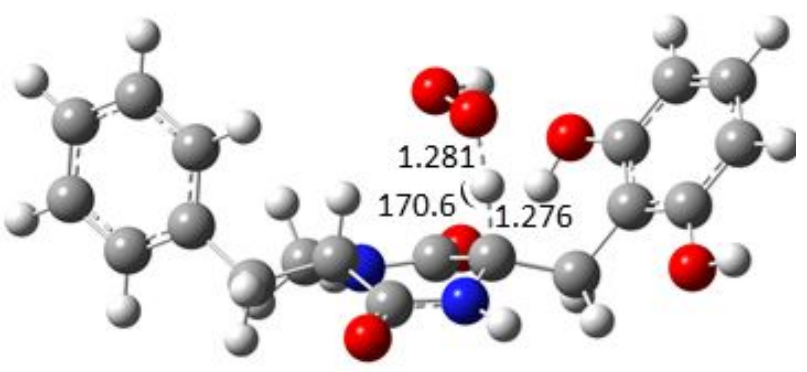

Figure S6: Optimized structure of the transition state (TS) for the first-HAT reaction towards $\mathrm{HOO}$ - radical calculated at the M05-2X/6-311++G(d,p) level of theory in PEA. Bond lengths are in $\AA$ and angles in degree. Values in parentheses consist in imaginary frequencies of TS 
STRUCTURE SOMO SOMO-1
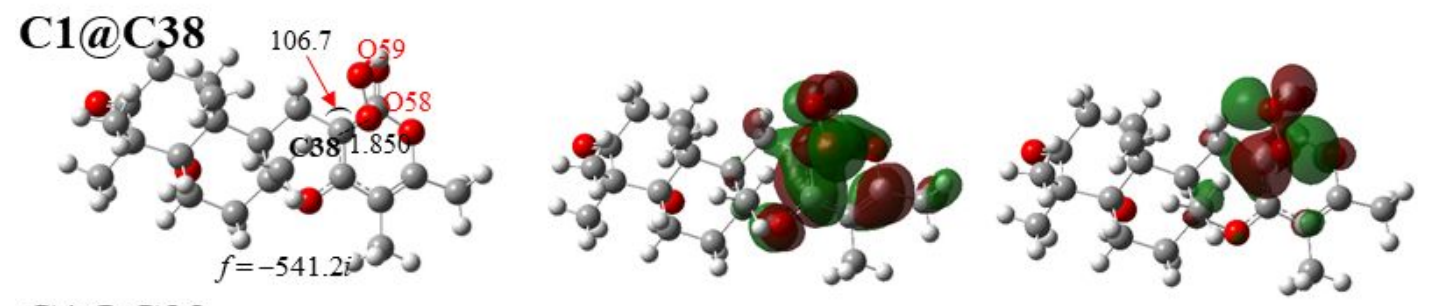

\section{C1@C39}

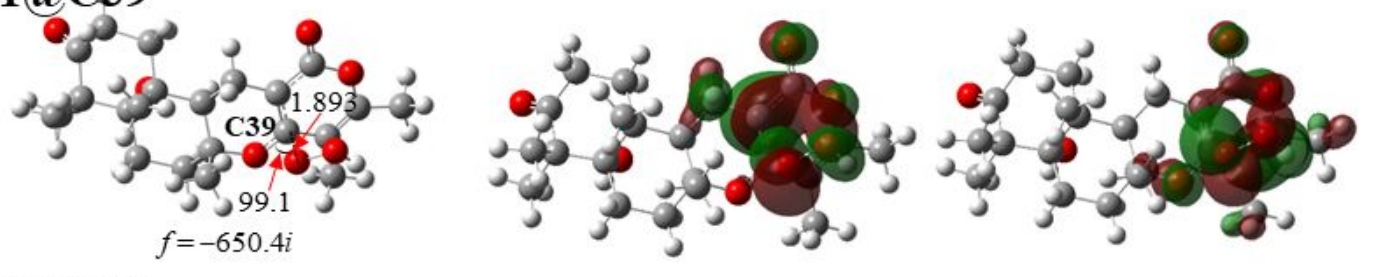

\section{C1@C46}
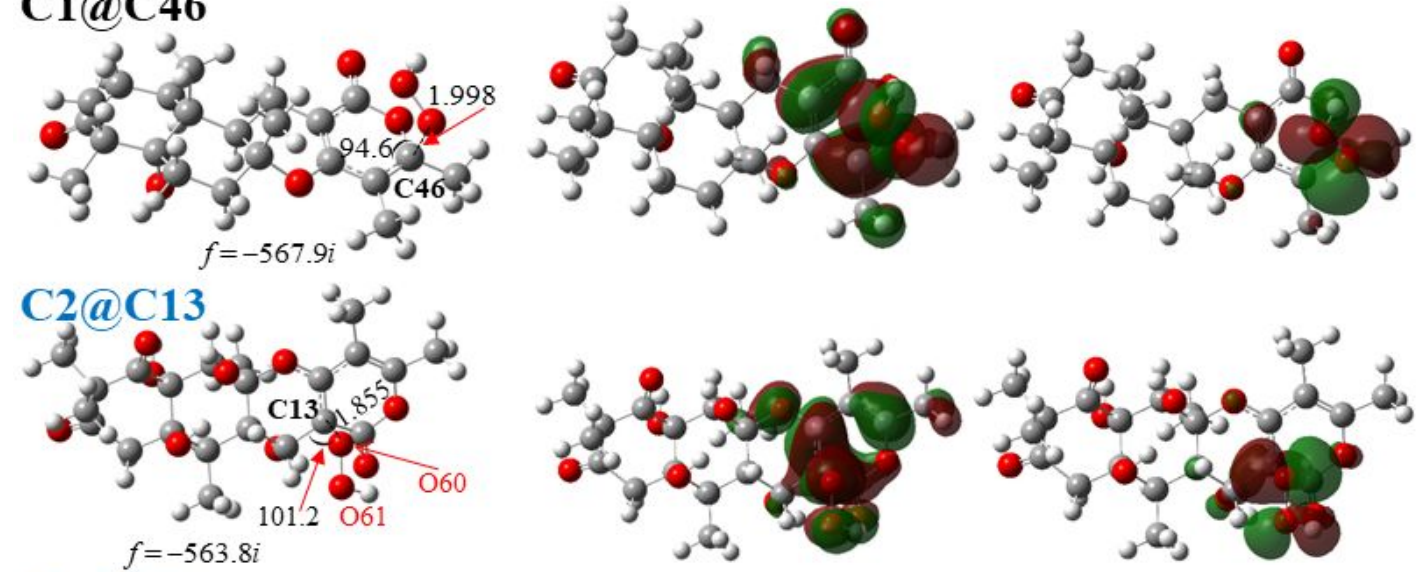

\section{C2@C34}
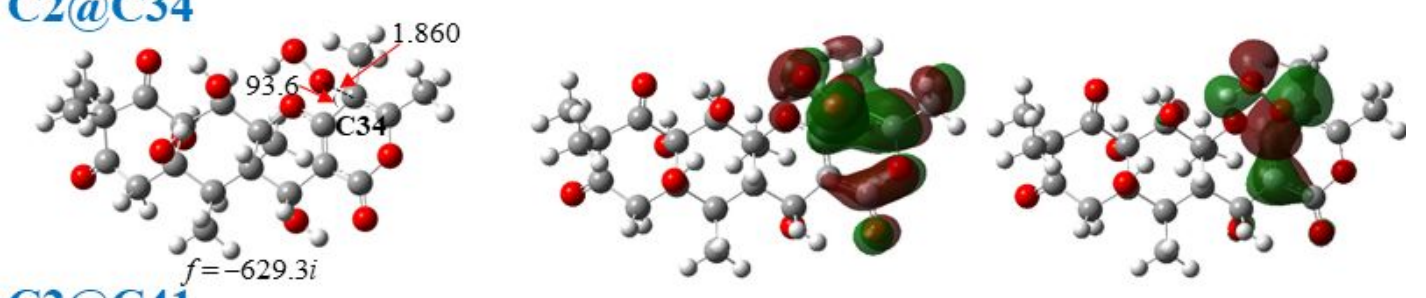

C2@C41
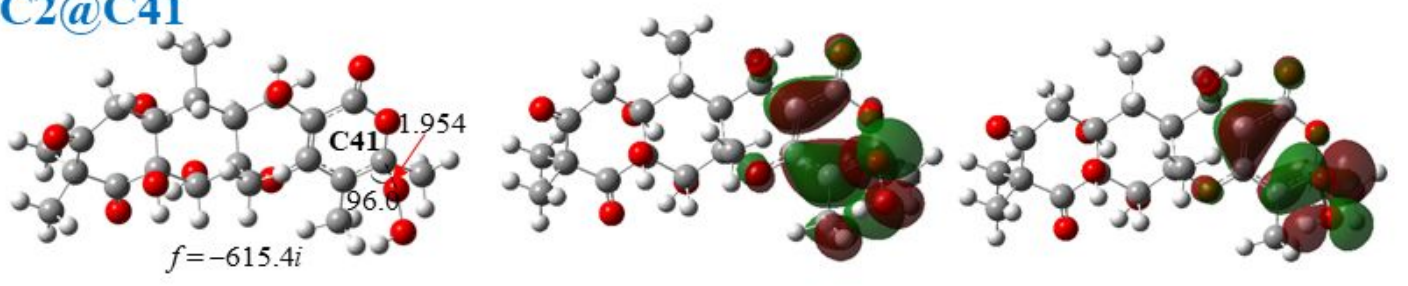


\section{STRUCTURE}
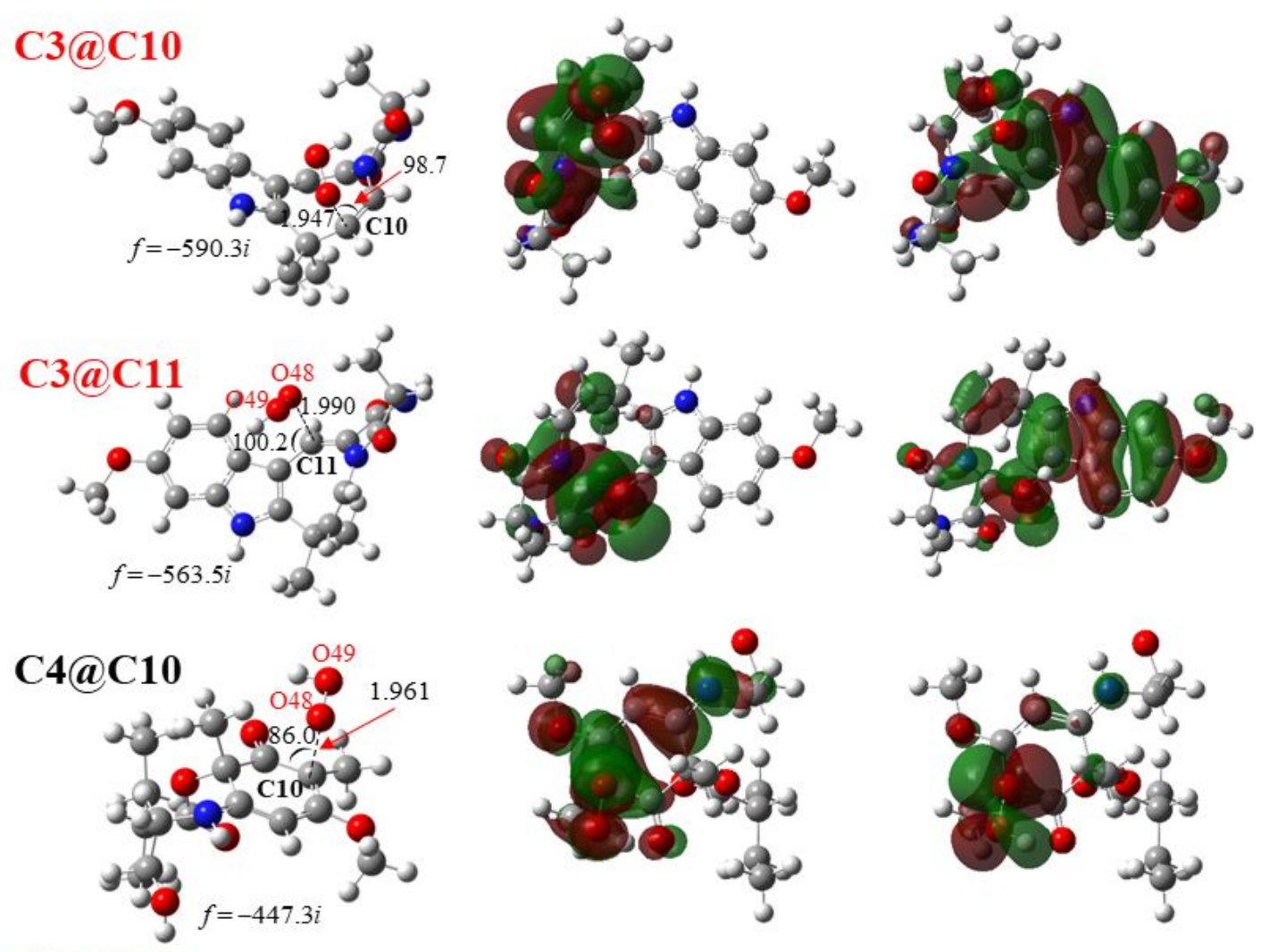

\section{C5@C20}
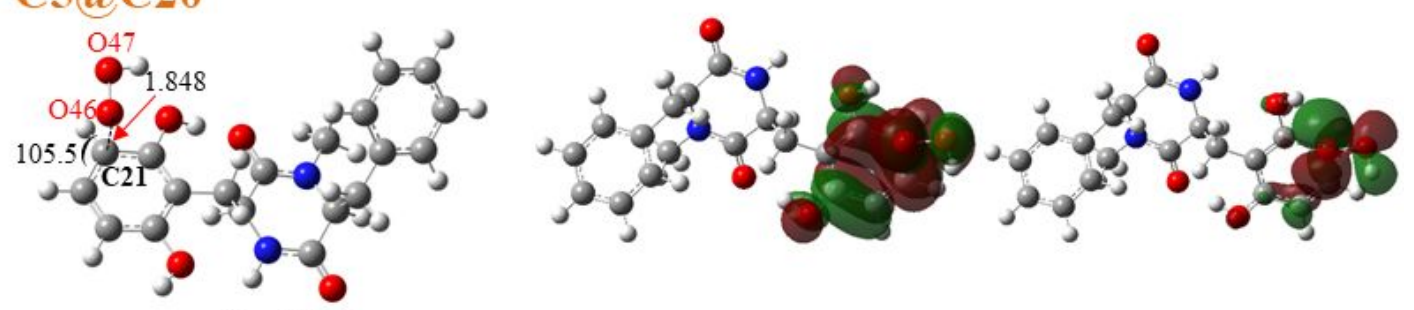

$f=-638.0 i$

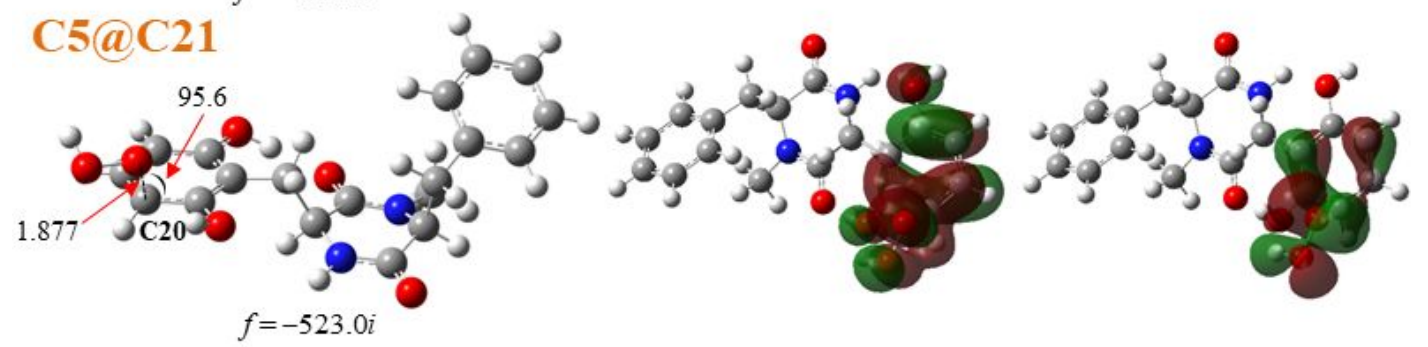

Figure S7: Optimized structures, singly occupied molecular orbital (SOMO) and SOMO-1 orbital distributions of the transition states (TS) of RAF reaction between $\mathbf{C 1}-\mathbf{C 5}$ with $\mathrm{HOO}$ - radical at different positions. Bond lengths are in $\AA$ and angles in degree. The imaginary frequencies ( $f$ ) are noted below each TS structure. All calculations were performed at the M05-2X/6$311++G(d, p)$ level of theory in the gas phase. 


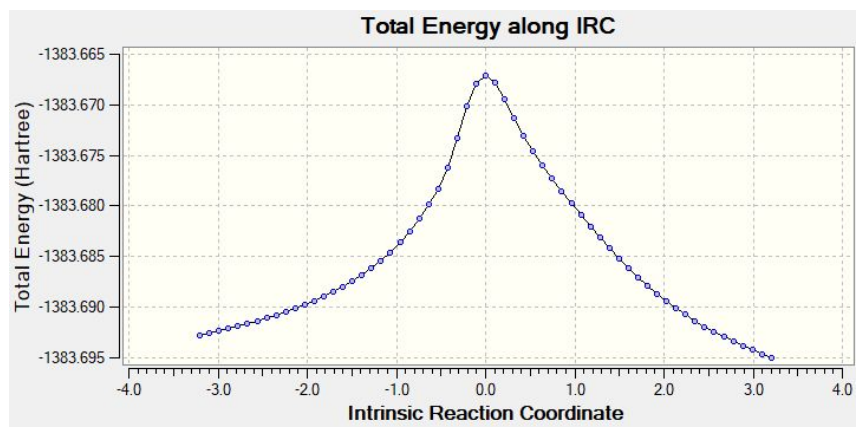

C1@C34H +OOH

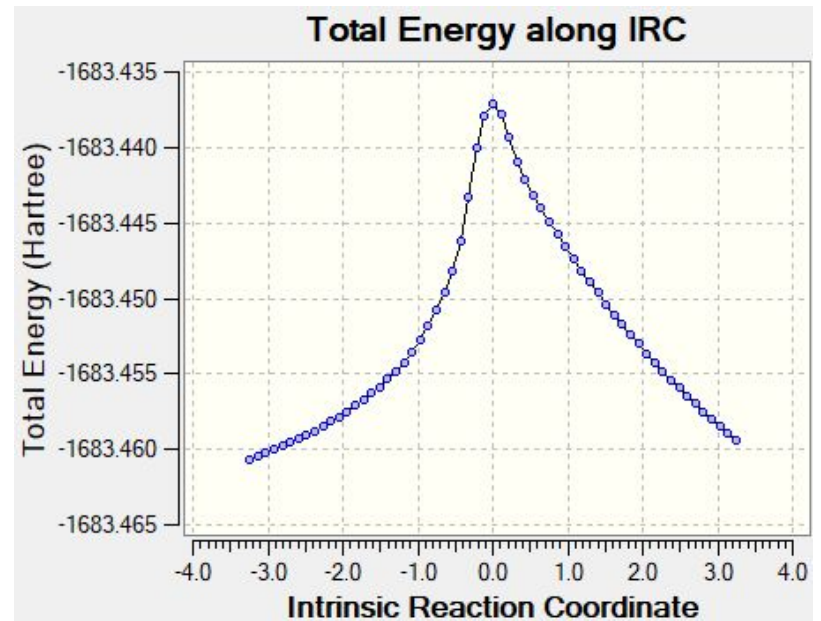

$\mathrm{C} 2 @ \mathrm{C} 17 \mathrm{H}+\mathrm{OOH}$

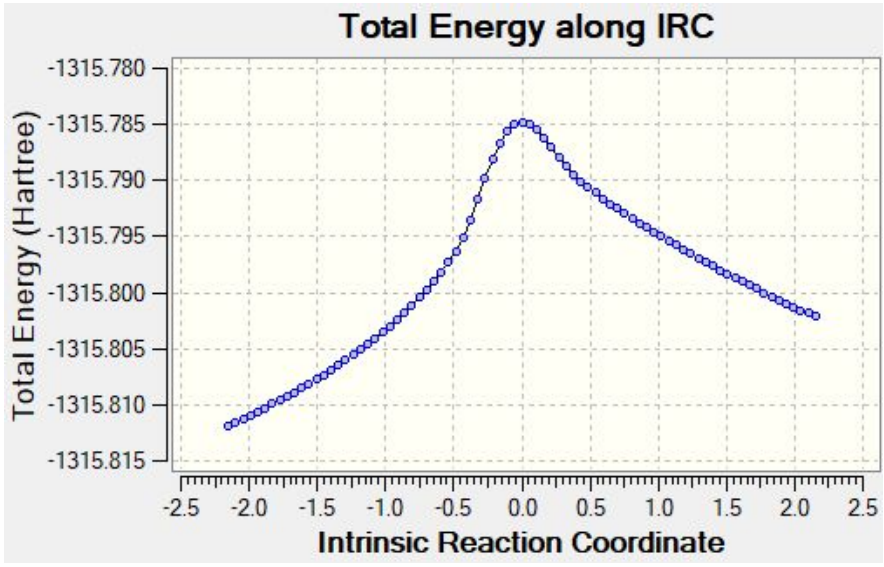

C3@C13H + OOH 

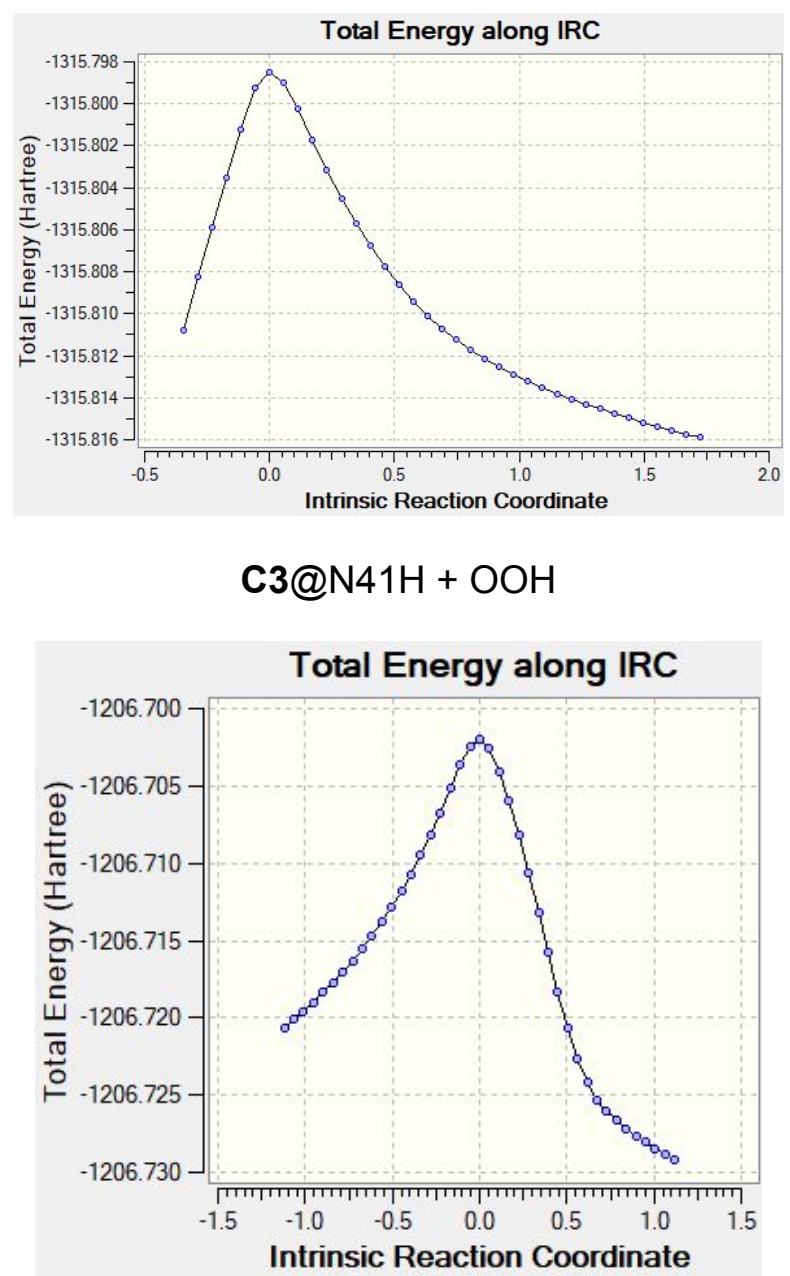

\section{C4@N6H + OOH}

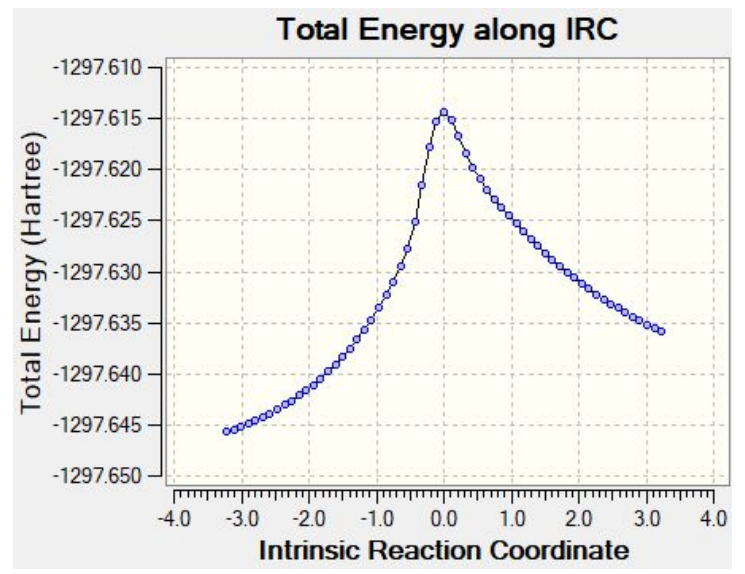

\section{C5@C8H + OOH}

Figure S8: Internal Reaction Coordinates (IRCs) of H-atom transfer (HAT) reactions calculated at the M05-2X/6-311++G(d,p) in the gas phase. 

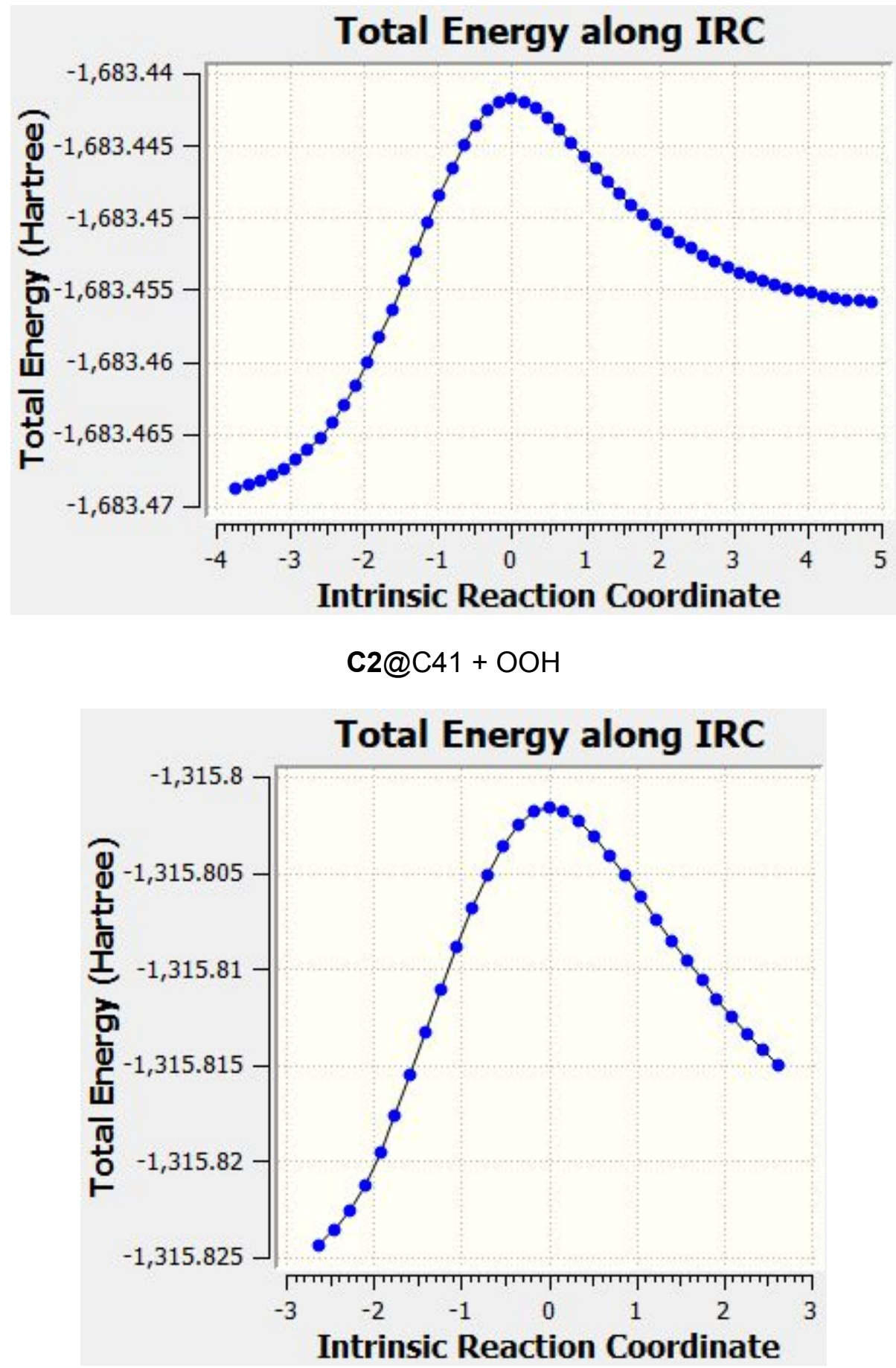

C3@C10+OOH 

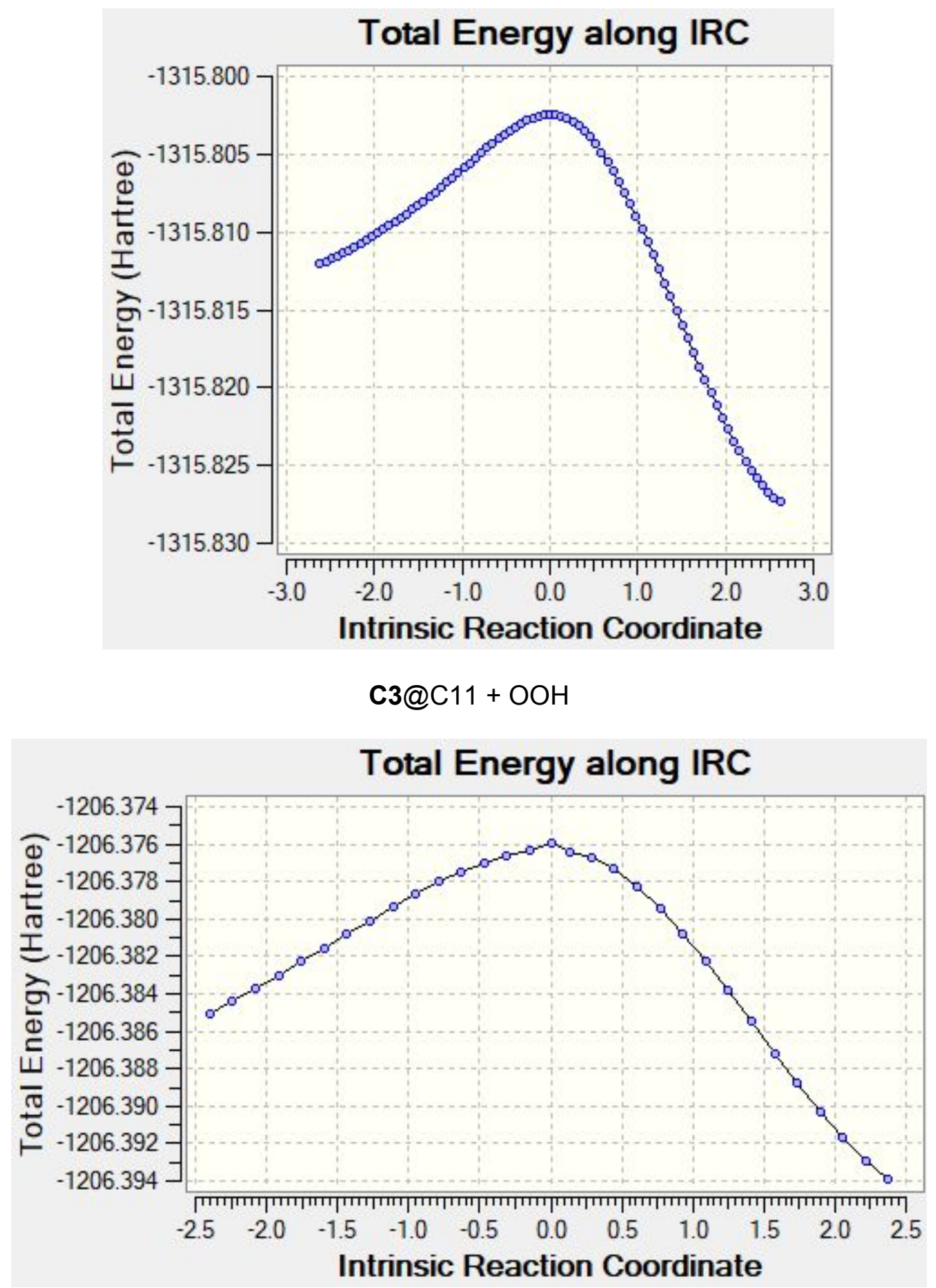

C4@C10+OOH 

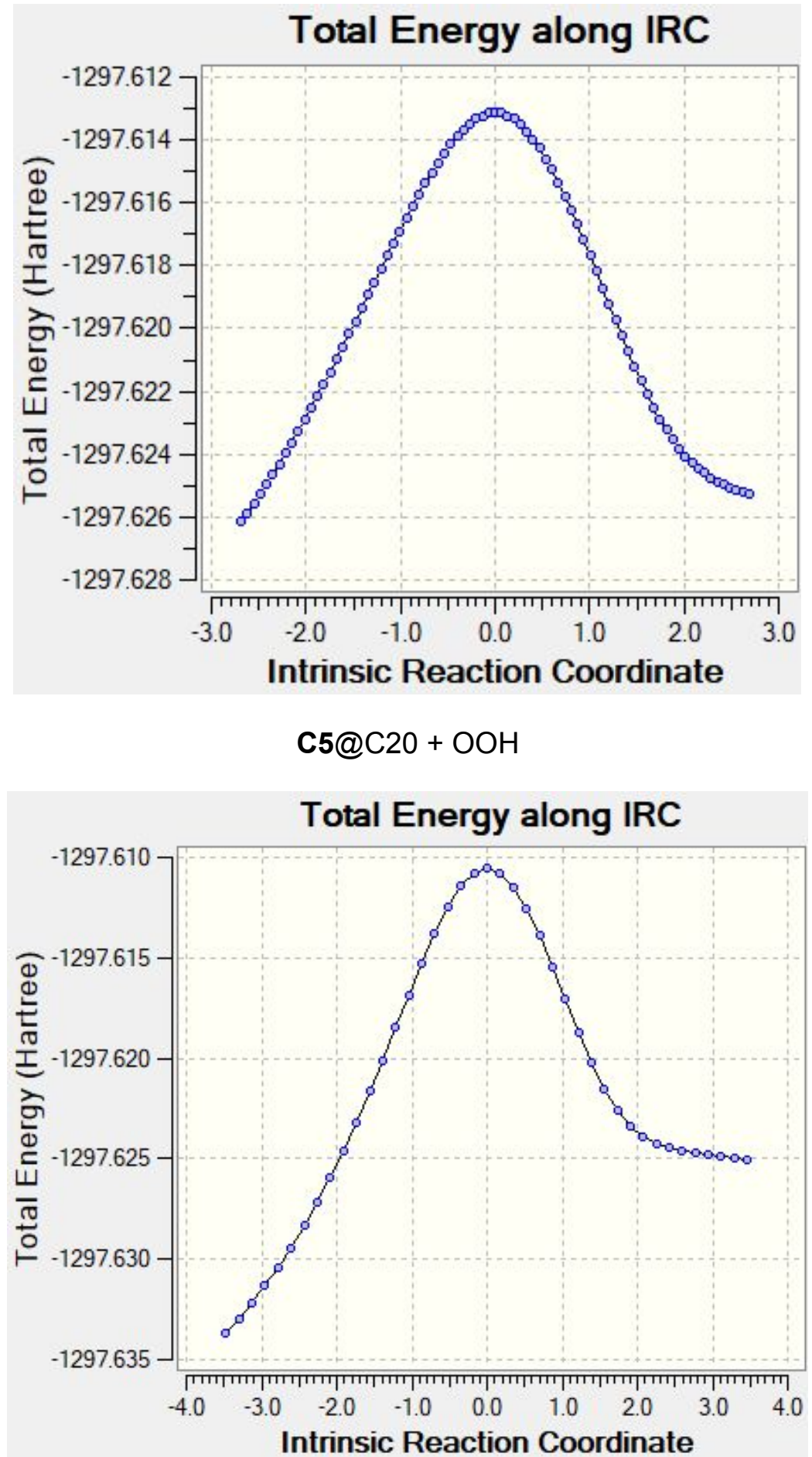

$$
\mathrm{C5} @ \mathrm{C} 2+\mathrm{OOH}
$$

Figure S9: Internal Reaction Coordinates (IRCs) of Radical Adduct Formation (RAF) reactions calculated at the M05-2X/6-311++G(d,p) in the gas phase. 

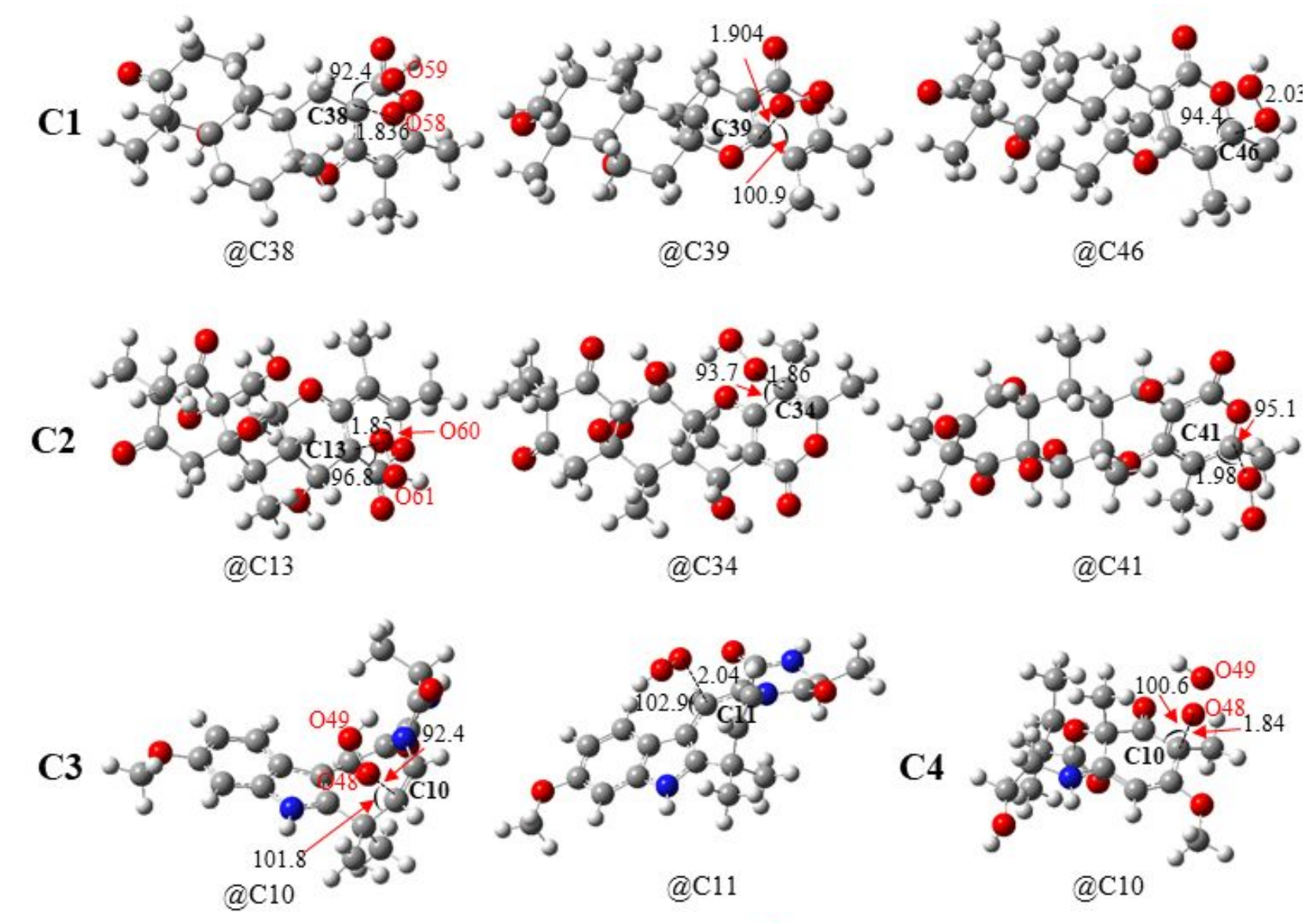

C5
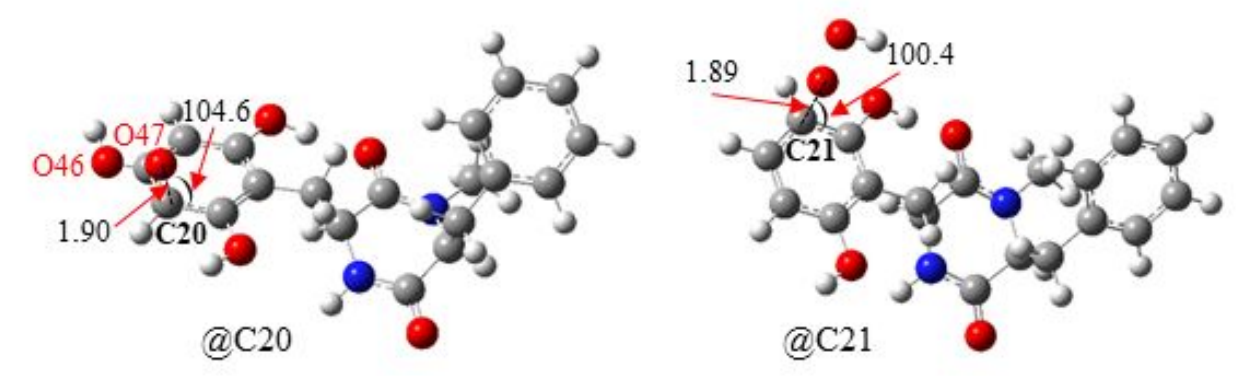

Figure S10: Optimized structure of the transition states (TSs) for RAF mechanism with $\mathrm{HOO} \cdot$ radical in the water. Bond lengths are in $\AA$ and angles in degree. All calculations were performed in the gas phase at the M05-2X/6-311++G(d,p) level of theory. 
C1
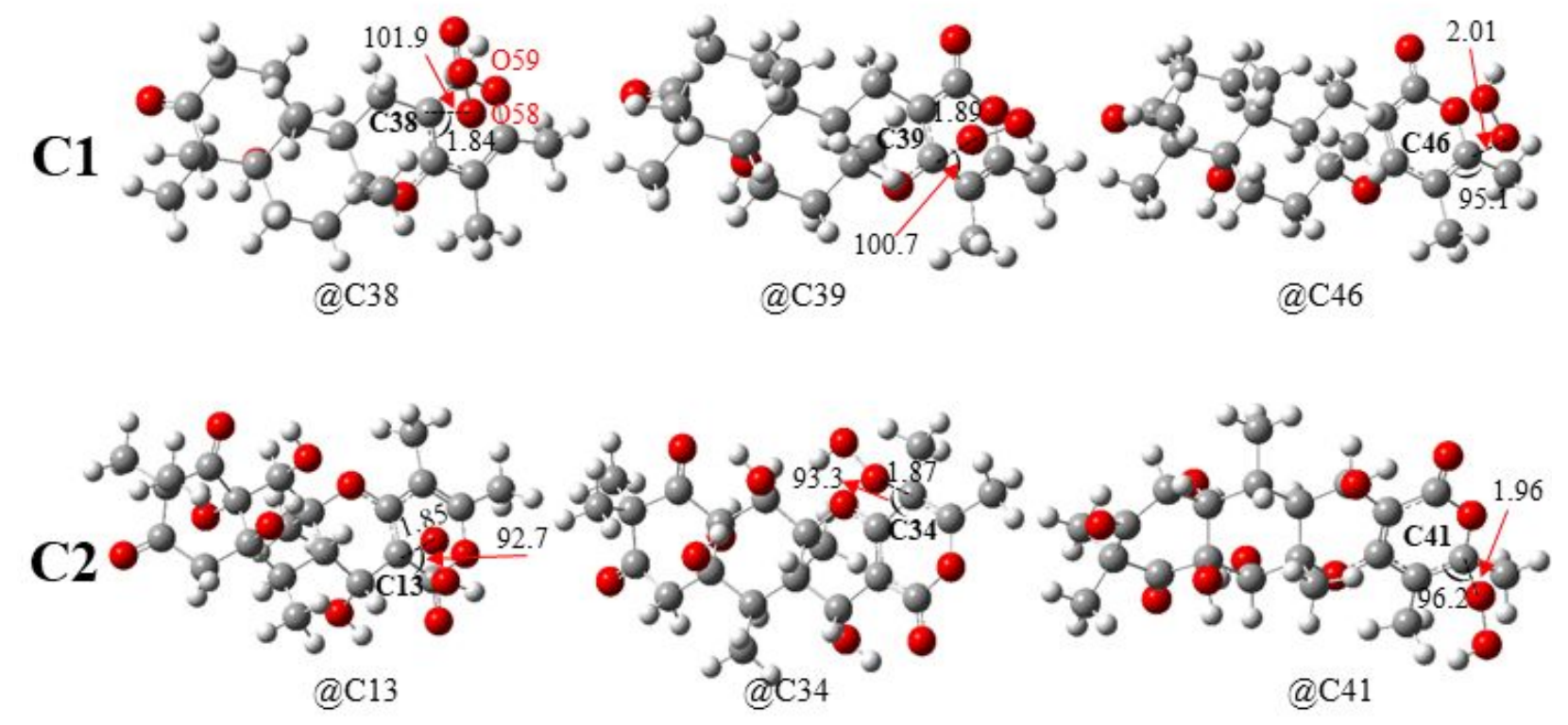

C3
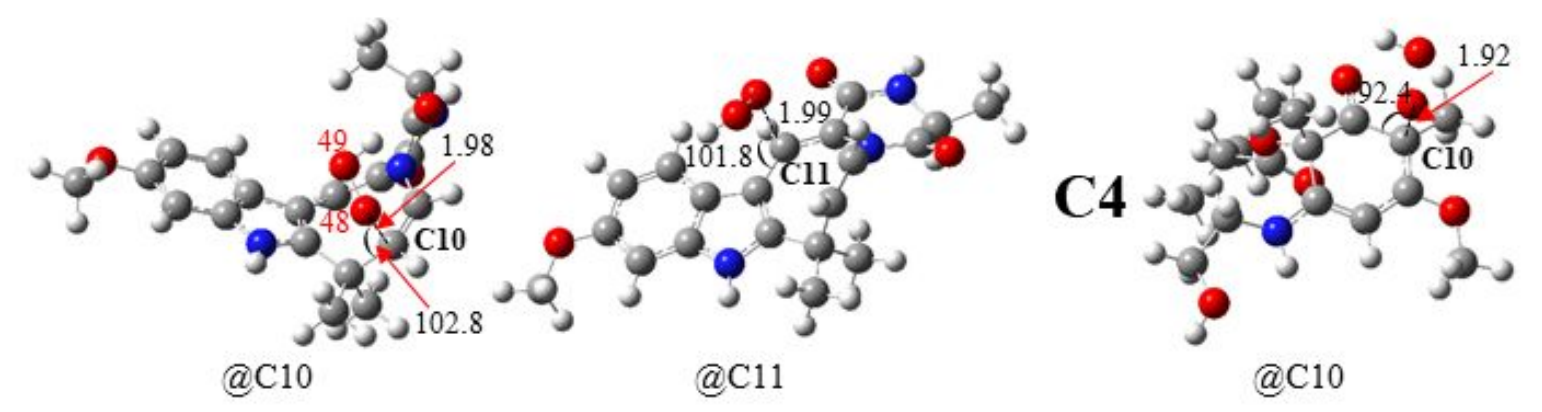

C5
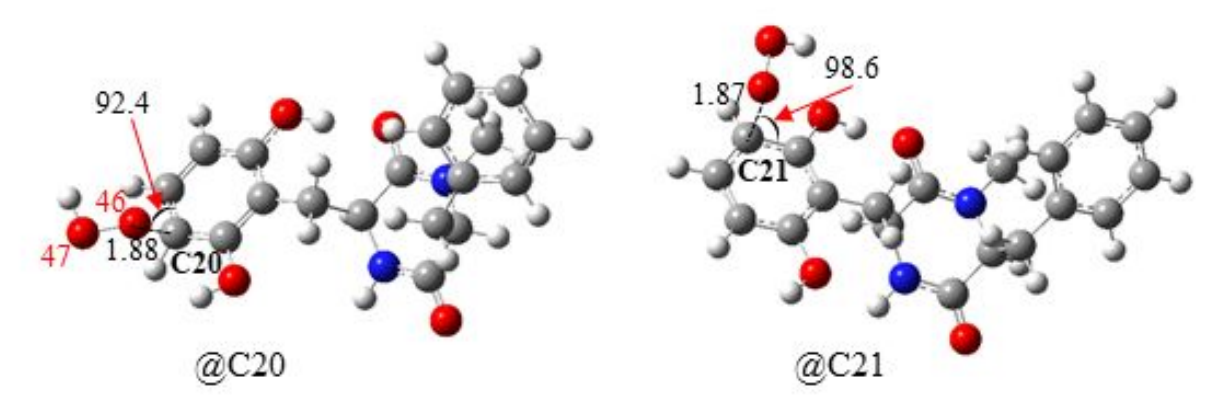

Figure S11: Optimized structure of the transition states (TSs) for RAF mechanism with $\mathrm{HOO}$ • radical in the PEA. Bond lengths are in $\AA$ and angles in degree. All calculations were performed in the gas phase at the M05-2X/6-311++G(d,p) level of theory. 
Table S1: BDE values (in kcal mol-1) characterizing two steps of double HT mechanism calculated in the gas phase, water and pentyl ethanoate (PEA) at the M05-2X/6-311++G(d,p)//

$M 05-2 X / 6-31+G(d)$ level of theory.

\begin{tabular}{|c|c|c|c|}
\hline $\begin{array}{l}\text { Compound / } \\
\text { Bond positions }\end{array}$ & GAS & WATER & PEA \\
\hline \multicolumn{4}{|c|}{ Ochraceopone F (C1) } \\
\hline $\mathrm{C} 1-\mathrm{C} 34 \mathrm{H}$ & 83.8 & 86.1 & 84.5 \\
\hline C1-C34H // C5-H & 46.0 & 45.3 & 45.1 \\
\hline $\mathrm{C} 1-\mathrm{C} 42 \mathrm{H}$ & 103.3 & 90.2 & 88.7 \\
\hline C1-C42H // C5-H & 83.8 & 85.6 & 82.7 \\
\hline C1-C5H & 95.5 & 95.5 & 95.1 \\
\hline \multicolumn{4}{|c|}{ Aspertetranone D (C2) } \\
\hline C2-C14H & 92.5 & 94.9 & 93.2 \\
\hline $\mathrm{C} 2-\mathrm{C} 14 \mathrm{H} / / \mathrm{C} 35-\mathrm{H}$ & 68.2 & 70.6 & 68.9 \\
\hline $\mathrm{C} 2-\mathrm{C} 14 \mathrm{H} / / \mathrm{C} 38-\mathrm{H}$ & 64.6 & 64.6 & 63.8 \\
\hline C2-C14H // O1-H & 44.1 & 41.8 & 42.0 \\
\hline $\mathrm{C2}-\mathrm{C} 17 \mathrm{H}$ & 81.2 & 86.4 & 82.5 \\
\hline $\mathrm{C} 2-\mathrm{C} 17 \mathrm{H} / / \mathrm{O} 57-\mathrm{H}$ & 44.4 & 37.4 & 39.9 \\
\hline \multicolumn{4}{|c|}{ Cycloechinulin (C3) } \\
\hline $\mathrm{C} 3-\mathrm{C} 13 \mathrm{H}$ & 80.5 & 82.9 & 80.7 \\
\hline C3-N41H & 77.1 & 80.3 & 79.0 \\
\hline C3-N41H // C13-H & 76.6 & 71.4 & 73.4 \\
\hline \multicolumn{4}{|c|}{ Wasabidienone E (C4) } \\
\hline C4-C11H & 86.3 & 86.5 & 86.0 \\
\hline C4-C11H // C16-H & 55.1 & 57.7 & 56.2 \\
\hline C4-C11H // C20-H & 52.9 & 55.3 & 54.0 \\
\hline $\mathrm{C} 4-\mathrm{C} 11 \mathrm{H} / / \mathrm{O} 5-\mathrm{H}$ & 46.4 & 45.8 & 45.3 \\
\hline C4-C16H & 85.8 & 85.4 & 84.6 \\
\hline C4-C16H // C11-H & 55.6 & 58.9 & 57.5 \\
\hline C4-C16H //N6-H & 42.2 & 44.0 & 43.2 \\
\hline C4-C16H //O5-H & 38.3 & 41.5 & 39.4 \\
\hline C4-C17H & 84.0 & 87.6 & 85.4 \\
\hline C4-C17H // C16-H & 76.7 & 78.2 & 76.1 \\
\hline C4-C17H // C11-H & 68.0 & 70.4 & 68.5 \\
\hline C4-C17H // O5-H & 48.7 & 50.8 & 49.1 \\
\hline C4-C17H // N6-H & 46.6 & 50.1 & 48.5 \\
\hline $\mathrm{C} 4-\mathrm{C} 2 \mathrm{OH}$ & 90.8 & 94.6 & 91.7 \\
\hline $\mathrm{C} 4-\mathrm{C} 20 \mathrm{H} / / \mathrm{C} 11-\mathrm{H}$ & 55.9 & 52.1 & 53.0 \\
\hline $\mathrm{C} 4-\mathrm{C} 2 \mathrm{OH} / / \mathrm{N} 6-\mathrm{H}$ & 43.1 & 46.4 & 45.4 \\
\hline $\mathrm{C} 4-\mathrm{C} 2 \mathrm{OH} / / \mathrm{O} 5-\mathrm{H}$ & 31.6 & 28.8 & 28.4 \\
\hline C4-N6H & 74.9 & 79.4 & 77.7 \\
\hline
\end{tabular}




\begin{tabular}{|c|c|c|c|}
\hline C4-N6H // C16-H & 53.1 & 49.4 & 49.8 \\
\hline C4-N6H // C20-H & 64.2 & 63.9 & 63.4 \\
\hline C4-N6H // O5-H & 67.3 & 71.8 & 69.0 \\
\hline \multicolumn{4}{|l|}{ Mactanamide (C5) } \\
\hline C5-C7H & 81.0 & 84.5 & 82.2 \\
\hline C5-C7H // C8-H & 60.5 & 59.3 & 60.0 \\
\hline C5-C7H // C10-H & 50.6 & 53.3 & 51.0 \\
\hline $\mathrm{C5}-\mathrm{C} 8 \mathrm{H}$ & 74.5 & 79.4 & 76.3 \\
\hline C5-C8H // C7-H & 66.9 & 64.5 & 65.9 \\
\hline C5-C8H // C11-H & 55.3 & 54.6 & 54.4 \\
\hline C5-C11H & 86.5 & 88.7 & 86.8 \\
\hline C5-C11H // C8-H & 42.3 & 44.1 & 42.1 \\
\hline $\mathrm{C} 5-\mathrm{C} 13 \mathrm{H}$ & 92.8 & 95.6 & 93.3 \\
\hline C5-C13H // C8-H & 75.1 & 71.9 & 72.5 \\
\hline C5-C13H // C10-H & 66.6 & 66.3 & 66.4 \\
\hline
\end{tabular}


Table S2: Reaction enthalpies $\left(\Delta_{r} H^{0}\right)$ and standard Gibbs free energies $\left(\Delta_{r} G^{0}\right)$ (in kcal mol-1) of RAF reactions between $\mathbf{C} 1-\mathbf{C 5}$ and $\mathrm{HOO} \cdot$ radical calculated at the M05-2X/6-311++G(d,p) level of theory in the gas phase, water and pentyl ethanoate (PEA).

\begin{tabular}{|c|c|c|c|c|c|c|c|}
\hline \multirow{2}{*}{\multicolumn{2}{|c|}{$\begin{array}{l}\text { Compounds / } \\
\text { Bond positions }\end{array}$}} & \multicolumn{2}{|c|}{ GAS } & \multicolumn{2}{|c|}{ WATER } & \multicolumn{2}{|c|}{ PEA } \\
\hline & & $\Delta_{\mathrm{r}} H^{0}$ & $\Delta_{\mathrm{r}} \boldsymbol{G}^{0}$ & $\Delta_{\mathrm{r}} H^{0}$ & $\Delta_{\mathrm{r}} \boldsymbol{G}^{0}$ & $\Delta_{\mathrm{r}} H^{0}$ & $\Delta_{r} G^{0}$ \\
\hline \multirow{4}{*}{ C1 } & C38 & 0.7 & 12.4 & 6.7 & 18.5 & -0.4 & -0.4 \\
\hline & C39 & 1.4 & 14.0 & 5.3 & 17.9 & -0.4 & -0.4 \\
\hline & C41 & 0.4 & 12.7 & 2.0 & 13.9 & -0.4 & -0.4 \\
\hline & C46 & -17.0 & -4.4 & -13.5 & -1.3 & -0.4 & -0.4 \\
\hline \multirow{3}{*}{ C2 } & $\mathrm{C} 13$ & 0.3 & 11.5 & 5.7 & 18.0 & 6.1 & 17.7 \\
\hline & C34 & -0.5 & 10.9 & 3.2 & 15.8 & 4.8 & 16.8 \\
\hline & C41 & -15.3 & -3.6 & -12.1 & 0.3 & -10.6 & 2.1 \\
\hline \multirow{7}{*}{ C3 } & C6 & 2.1 & 14.1 & & & & \\
\hline & C8 & 15.7 & 27.3 & & & & \\
\hline & C10 & -18.1 & -6.1 & -15.0 & -3.3 & -12.4 & -0.6 \\
\hline & C11 & -18.9 & -5.8 & -15.3 & -2.1 & -13.6 & -0.1 \\
\hline & C18 & 1.2 & 12.8 & & & & \\
\hline & C19 & 0.4 & 12.4 & & & & \\
\hline & $\mathrm{C} 21$ & 5.1 & 16.9 & & & & \\
\hline \multirow{3}{*}{ C4 } & C9 & -11.5 & 4.1 & 1.5 & 14.5 & 8.0 & 21.1 \\
\hline & C10 & -12.2 & 0.3 & -3.1 & 11.0 & -3.5 & 10.0 \\
\hline & $\mathrm{C} 12$ & $\begin{array}{l}-7.8 \\
\end{array}$ & 4.0 & & & & \\
\hline \multirow{8}{*}{ C5 } & C15 & 7.9 & 19.2 & & & & \\
\hline & C19 & 8.6 & 20.0 & & & & \\
\hline & $C 20$ & 2.7 & 14.1 & 6.1 & 17.6 & 8.1 & 19.5 \\
\hline & $\mathrm{C} 21$ & 3.5 & 15.7 & 5.6 & 17.1 & 7.5 & 19.5 \\
\hline & $\mathrm{C} 22$ & 6.0 & 18.4 & & & & \\
\hline & $\mathrm{C} 23$ & 7.4 & 18.5 & & & & \\
\hline & $\mathrm{C} 24$ & 7.8 & 19.5 & & & & \\
\hline & $\mathrm{C} 25$ & 7.1 & 17.8 & & & & \\
\hline
\end{tabular}


Table S3: Intrinsic properties characterizing for double SET-PT process (IP and PDE, in kcal $\mathrm{mol}^{-1}$ ) calculated in the gas phase, water and PEA using the M05-2X/6-311++G(d,p)//M05-2X/6$31+G(d)$ level of theory.

\begin{tabular}{|l|c|c|c|}
\hline $\begin{array}{l}\text { Compound I } \\
\text { Proton donating positions }\end{array}$ & GAS & WATER & PEA \\
\hline Ochraceopone F (C1) & 179.0 & 124.4 & 132.9 \\
\hline C1-IP1 & 218.8 & 6.1 & 10.0 \\
\hline C1-C34H-PDE1 & 222.9 & 10.2 & 14.3 \\
\hline C1-C42H-PDE1 & 230.6 & 15.5 & 20.7 \\
\hline C1-C5H-PDE1 & 142.8 & 88.0 & 96.8 \\
\hline C1-C34H-IP2 & 317.4 & 94.2 & 105.7 \\
\hline C1-C34H/C19H-PDE2 & 187.9 & 129.6 & 140.2 \\
\hline Aspertetranone D (C2) & 223.9 & 13.9 & 16.2 \\
\hline C2-IP1 & 235.4 & 25.3 & 26.8 \\
\hline C2-C36H-PDE1 & 382.2 & 131.9 & 140.2 \\
\hline C2-O1H-PDE1 & 159.5 & 98.8 & 111.2 \\
\hline C2-O5H-PDE1 & 180.1 & 90.8 & 97.8 \\
\hline C2-C36H-IP2 & 154.3 & 102.8 & 110.7 \\
\hline C2-C36H/C17H-PDE2 & 236.7 & 19.0 & 24.2 \\
\hline Cycloechinulin (C3) & 252.3 & 36.3 & 41.4 \\
\hline C3-IP1 & 236.9 & 21.9 & 26.9 \\
\hline C3-C13H-PDE1 & 146.6 & 92.0 & 100.3 \\
\hline C3-C22H-PDE1 & 217.9 & 5.5 & 9.6 \\
\hline C3-N41H-PDE1 & 151.2 & 99.2 & 106.4 \\
\hline C3-C13H-IP2 & 248.6 & 30.5 & 36.7 \\
\hline C3-C13H/C43H-PDE2 & 246.9 & 32.8 & 37.5 \\
\hline Wasabidienone E (C4) & 253.6 & 39.8 & 43.8 \\
\hline C4-IP1 & 277.5 & 24.6 & 29.8 \\
\hline C4-C16H-PDE1 & 251.3 & 72.8 & 83.3 \\
\hline C4-C17H-PDE1 & 212.9 & 1.2 & 5.0 \\
\hline C4-C20H-PDE1 & 177.0 & 119.9 & 129.7 \\
\hline C4-N6H-PDE1 & 218.1 & 11.9 & 14.1 \\
\hline C4-C16H-IP2 & 211.7 & 6.8 & 8.2 \\
\hline C4-C16H/C18H-PDE2 & 373.6 & 358.8 & 359.4 \\
\hline Mactanamide (C5) & 18.0 & -242.1 & -227.1 \\
\hline C5-IP1 & \multicolumn{2}{|l|}{} \\
\hline C5-C7H-PDE1 & \multicolumn{3}{|l|}{} \\
\hline C5-C8H-PDE1 & \multicolumn{3}{|l|}{} \\
\hline C5-C8H-IP2 & \multicolumn{3}{|l|}{} \\
\hline C5-C8H/C19H-PDE2 & \multicolumn{3}{|l|}{} \\
\hline
\end{tabular}


Table S4: Intrinsic properties characterizing for double SPL-ET process (PA and ETE, in kcal $\mathrm{mol}^{-1}$ ) calculated in the gas phase, water and PEA solvent using the M05-2X/6-311++G(d,p)//

M05-2X/6-31+G(d) level of theory.

\begin{tabular}{|c|c|c|c|}
\hline $\begin{array}{l}\text { Compound / } \\
\text { Proton donating positions }\end{array}$ & GAS & WATER & PEA \\
\hline \multicolumn{4}{|l|}{ Ochraceopone F (C1) } \\
\hline C1-C9H-PA1 & 385.1 & 91.5 & 112.6 \\
\hline C1-C5H-PA1 & 370.7 & 83.2 & 104.2 \\
\hline C1-C9H-ETE1 & 28.5 & 54.4 & 45.8 \\
\hline C1-C5H-ETE1 & 41.2 & 62.3 & 53.2 \\
\hline C1-C5H/C9H-PA2 & 369.7 & 79.5 & 102.6 \\
\hline C1-C5H/C9H-ETE2 & -1.2 & 18.3 & 9.2 \\
\hline \multicolumn{4}{|l|}{ Aspertetranone $\mathrm{D}(\mathrm{C} 2)$} \\
\hline C2-C49H-PA1 & 341.9 & 54.3 & 74.9 \\
\hline C2-O1H-PA1 & 332.5 & 41.0 & 66.0 \\
\hline C2-O5H-PA1 & 330.7 & 38.4 & 64.2 \\
\hline C2-O9H-PA1 & 332.5 & 41.0 & 66.0 \\
\hline C2-C49H-ETE1 & 58.7 & 79.1 & 71.0 \\
\hline C2-O1H-ETE1 & 93.5 & 117.5 & 104.5 \\
\hline C2-O5H-ETE1 & 84.1 & 112.5 & 96.4 \\
\hline C2-O9H-ETE1 & 93.5 & 117.5 & 104.5 \\
\hline $\mathrm{C} 2-\mathrm{O} 5 \mathrm{H} / \mathrm{C} 16 \mathrm{H}-\mathrm{PA} 2$ & 309.7 & 18.5 & 44.1 \\
\hline C2-O5H/C16H-ETE2 & 71.4 & 73.3 & 71.4 \\
\hline \multicolumn{4}{|l|}{ Cycloechinulin (C3) } \\
\hline C3-C13H-PA1 & 349.9 & 52.7 & 78.3 \\
\hline C3-C15H-PA1 & 343.1 & 43.0 & 70.8 \\
\hline C3-C14H-PA1 & 331.4 & 43.1 & 63.9 \\
\hline C3-N41H-PA1 & 274.7 & 43.0 & 63.7 \\
\hline C3-C13H-ETE1 & 44.6 & 74.6 & 61.0 \\
\hline C3-C15H-ETE1 & 43.0 & 75.8 & 61.1 \\
\hline C3-C14H-ETE1 & 59.8 & 81.6 & 73.7 \\
\hline C3-N41H-ETE1 & 116.4 & 81.7 & 73.8 \\
\hline C3-N41H/C13H-PA2 & 326.6 & 35.6 & 57.1 \\
\hline C3-N41H/C14H-PA2 & 347.3 & 57.4 & 78.8 \\
\hline C3-N41H/C15H-PA2 & 345.1 & 54.4 & 75.9 \\
\hline C3-N41H/C13H-ETE2 & 64.1 & 80.2 & 74.9 \\
\hline C3-N41H/C14H-ETE2 & 64.8 & 80.2 & 74.9 \\
\hline \multicolumn{4}{|l|}{ Wasabidienone E (C4) } \\
\hline C4-N6H-PA1 & 332.7 & 38.6 & 62.3 \\
\hline C4-O5H-PA1 & 331.1 & 37.0 & 60.5 \\
\hline C4-N6H-ETE1 & 58.2 & 85.8 & 74.3 \\
\hline
\end{tabular}




\begin{tabular}{|l|c|c|c|} 
C4-O5H-ETE1 & 59.8 & 87.3 & 76.1 \\
\hline C4-O5H/C16H-PA2 & 330.2 & 36.2 & 59.8 \\
\hline C4-O5H/C16H-ETE2 & 34.5 & 59.1 & 48.4 \\
\hline Mactanamide (C5) & 335.2 & 43.2 & 68.4 \\
\hline C5-O4H-PA1 & 331.8 & 39.4 & 64.1 \\
\hline C5-O3H-PA1 & 340.5 & 53.9 & 75.6 \\
\hline C5-C8H-PA1 & 68.0 & 94.3 & 82.8 \\
\hline C5-O4H-ETE1 & 66.9 & 96.0 & 83.0 \\
\hline C5-O3H-ETE1 & 48.1 & 72.8 & 62.3 \\
\hline C5-C8H-ETE1 & 317.4 & 25.0 & 47.2 \\
\hline C5-O3H/C8H-PA2 & 55.1 & 75.6 & 66.3 \\
\hline C5-O3H/C8H-ETE2 & &
\end{tabular}


Table S5: Excited energies (eV), absorption wavelength $(\mathrm{nm})$, oscillator strength $(f)$ and transition interpretation for some lowest excited states of compounds C1-C5 Calculated by TDDFT at the M05-2X/6-31+G(d) Level of Theory in Water.

\begin{tabular}{|c|c|c|c|c|c|c|}
\hline \multirow[t]{2}{*}{$\mathbf{C p}$} & \multicolumn{5}{|c|}{ Water } & \multirow[b]{2}{*}{$\begin{array}{l}\text { Exp. Abs/ } \\
\text { nm }\end{array}$} \\
\hline & $\mathbf{S}$ & $\mathrm{E} / \mathrm{eV}$ & $\begin{array}{l}\text { Abs/ } \\
\text { nm }\end{array}$ & $f$ & $\begin{array}{l}\text { Transition/ } \\
\text { (Contribution/\%) }\end{array}$ & \\
\hline \multirow[t]{4}{*}{ C1 } & 1 & 4.17 & 297.3 & 0.0002 & $\mathrm{H}-1 \rightarrow \mathrm{L}+1(81.7)$ & \multirow[t]{2}{*}{287} \\
\hline & 2 & 4.67 & 265.4 & 0.3228 & $\mathrm{H} \rightarrow \mathrm{L}(97.0)$ & \\
\hline & 4 & 6.06 & 204.6 & 0.0843 & $\mathrm{H}-2 \rightarrow \mathrm{L}(76.7)$ & \multirow{2}{*}{207} \\
\hline & 6 & 6.33 & 195.8 & 1.1125 & $\begin{array}{l}\mathrm{H} \rightarrow \mathrm{L}+5(56.2) \\
\mathrm{H} \rightarrow \mathrm{I}+4(199)\end{array}$ & \\
\hline \multirow[t]{4}{*}{ C2 } & 1 & 4.03 & 308.2 & 0.0000 & $\begin{array}{l}\mathrm{H}-2 \rightarrow \mathrm{L}+1(38.1) \\
\mathrm{H}-1 \rightarrow \mathrm{L}+1(44.1)\end{array}$ & \multirow[t]{4}{*}{289} \\
\hline & 2 & 4.29 & 288.7 & 0.0004 & $\begin{array}{l}\mathrm{H}-2 \rightarrow \mathrm{L}+2(65.1) \\
\mathrm{H}-1 \rightarrow \mathrm{L}+2(24.5)\end{array}$ & \\
\hline & 3 & 4.69 & 264.2 & 0.3287 & $\mathrm{H} \rightarrow \mathrm{L}(96.3)$ & \\
\hline & 7 & 6.19 & 200.2 & 1.0084 & $\begin{array}{l}\mathrm{H}-3 \rightarrow \mathrm{L}(37.7) \\
\mathrm{H} \rightarrow \mathrm{L}+3(25.0) \\
\mathrm{H} \rightarrow \mathrm{L}+4(28.6)\end{array}$ & \\
\hline \multirow[t]{12}{*}{$\mathbf{C 3}$} & 1 & 4.00 & 309.8 & 0.3420 & $\mathrm{H} \rightarrow \mathrm{L}(81.8)$ & \multirow{12}{*}{$\begin{array}{l}378 \\
302 \\
266 \\
233 \\
216\end{array}$} \\
\hline & 2 & 4.71 & 263.0 & 0.3366 & $\mathrm{H}-1 \rightarrow \mathrm{L}(25.2)$ & \\
\hline & & & & & $\mathrm{H} \rightarrow \mathrm{L}+1(23.4)$ & \\
\hline & & & & & $\mathrm{H}-2 \rightarrow \mathrm{L}(17.5)$ & \\
\hline & 4 & 5.06 & 244.9 & 0.1190 & $\mathrm{H}-1 \rightarrow \mathrm{L}(28.5)$ & \\
\hline & & & & & $\begin{array}{l}\mathrm{H}-2 \rightarrow \mathrm{L}(28.4) \\
\mathrm{H}-1 \rightarrow \mathrm{L}+1(13.2)\end{array}$ & \\
\hline & 6 & 5.33 & 232.5 & 0.1619 & $\mathrm{H} \rightarrow \mathrm{L}+2(26.4)$ & \\
\hline & & & & & $\begin{array}{l}\mathrm{H} \rightarrow \mathrm{L}+1(14.5) \\
\mathrm{H}-1 \rightarrow \mathrm{L}+1(8.7)\end{array}$ & \\
\hline & 10 & 5.74 & 215.9 & 0.5024 & $\mathrm{H}-2 \rightarrow \mathrm{L}+1(33.4)$ & \\
\hline & & & & & $\mathrm{H}-2 \rightarrow \mathrm{L}+2(15.2)$ & \\
\hline & & & & & $\mathrm{H} \rightarrow \mathrm{L}+2(13.2)$ & \\
\hline & & & & & $\mathrm{H} \rightarrow \mathrm{L}+1(10.9)$ & \\
\hline \multirow[t]{6}{*}{$\mathrm{C} 4$} & 1 & 3.38 & 366.4 & 0.3278 & $\mathrm{H} \rightarrow \mathrm{L}(98.0)$ & 416 \\
\hline & 3 & 5.32 & 233.1 & 0.7832 & $\mathrm{H}-1 \rightarrow \mathrm{L}(65.5)$ & \multirow{3}{*}{235} \\
\hline & & & & & $\mathrm{H} \rightarrow \mathrm{L}+1(15.2)$ & \\
\hline & & & & & $\mathrm{H} \rightarrow \mathrm{L}+3(10.3)$ & \\
\hline & 9 & 6.55 & 189.4 & 0.0359 & $\mathrm{H} \rightarrow \mathrm{L}+6(46.4)$ & 206 \\
\hline & & & & & $\begin{array}{l}\mathrm{H} \rightarrow \mathrm{L}+10(21.2) \\
\mathrm{H} \rightarrow \mathrm{L}+4(9.9)\end{array}$ & \\
\hline \multirow[t]{7}{*}{ C5 } & 1 & 5.26 & 235.6 & 0.0849 & $\mathrm{H} \rightarrow \mathrm{L}+2(69.9)$ & 259 \\
\hline & & & & & $\mathrm{H}-1 \rightarrow \mathrm{L}+5$ (16.7) & \\
\hline & 6 & 6.09 & 203.4 & 0.2403 & $\mathrm{H}-2 \rightarrow \mathrm{L}(53.3)$ & \multirow[t]{5}{*}{203} \\
\hline & & & & & $\mathrm{H}-3 \rightarrow \mathrm{L}+1$ (13.4) & \\
\hline & 8 & 6.22 & 199.3 & 1.2505 & $\mathrm{H}-1 \rightarrow \mathrm{L}+2(51.0)$ & \\
\hline & 11 & 6.51 & 190.5 & 0.7710 & $\begin{array}{l}\mathrm{H} \rightarrow \mathrm{L}+5(36.0) \\
\mathrm{H}-2 \rightarrow \mathrm{L}+1(35.4)\end{array}$ & \\
\hline & & & & & $\mathrm{H}-3 \rightarrow \mathrm{L}(34.3)$ & \\
\hline
\end{tabular}

